\title{
A Fractional-Order Model for Zika Virus Infection with Multiple Delays
}

\author{
R. Rakkiyappan $\mathbb{D}^{1},{ }^{1}$ V. Preethi Latha, ${ }^{1}$ and Fathalla A. Rihan ${ }^{2}{ }^{2}$ \\ ${ }^{1}$ Department of Mathematics, Bharathiar University, Coimbatore-641 046, Tamil Nadu, India \\ ${ }^{2}$ Department of Mathematical Sciences, College of Science, UAE University, Al Ain 15551, UAE \\ Correspondence should be addressed to Fathalla A. Rihan; frihan@uaeu.ac.ae
}

Received 19 August 2019; Revised 21 October 2019; Accepted 31 October 2019; Published 14 November 2019

Guest Editor: Serdar Çiçek

Copyright (C) 2019 R. Rakkiyappan et al. This is an open access article distributed under the Creative Commons Attribution License, which permits unrestricted use, distribution, and reproduction in any medium, provided the original work is properly cited.

\begin{abstract}
Time delays and fractional order play a vital role in biological systems with memory. In this paper, we propose an epidemic model for Zika virus infection using delay differential equations with fractional order. Multiple time delays are incorporated in the model to consider the latency of the infection in a vector and the latency of the infection in the infected host. We investigate the necessary and sufficient conditions for stability of the steady states and Hopf bifurcation with respect to three time delays $\tau_{1}$, $\tau_{2}$, and $\tau_{3}$. The model undergoes a Hopf bifurcation at the threshold parameters $\tau_{1}^{*}, \tau_{2}^{*}$, and $\tau_{3}^{*}$. Some numerical simulations are given to show the effectiveness of obtained results. The numerical simulations confirm that combination of fractional order and time delays in the epidemic model effectively enriches the dynamics and strengthens the stability condition of the model.
\end{abstract}

\section{Introduction}

Zika infection is a mosquito-borne disease, transmitted to humans through the bite of an infected Aedes mosquito. It was first discovered in Uganda in 1947 in rhesus monkey. The first human cases were reported in Nigeria in 1954. Zika was thought to cause mild symptoms in humans, including mild fever, skin rashes, conjunctivitis, muscle and joint pain, and headache, which lasts for three to twelve days normally. However, the World Health Organization (WHO) has concluded that Zika virus infection during pregnancy is also a cause of congenital brain abnormalities, including microcephaly [1]. Moreover, Zika virus is a trigger of Guillain-Barre syndrome [2]. There is no doubt that mathematical modeling of Zika infection plays an important role in gaining understanding of transmission of disease and to predict the behaviour of any outbreak [3, 4].

Recently, mathematical modeling of dynamics of infectious diseases, using differential equations with memory (time-delay terms or fractional orders), has attracted much attention of many researchers (see, e.g., [5] and references therein). Time delay in models of population dynamics and in particular in macroscopic models of the immune response are natural and common [6]. Naturally, time delay or memory is an unavoidable factor in dynamics of most reallife phenomena. Time delay has influence on dynamical behaviours of biological systems in various aspects. Therefore, considering time delays in the investigation of biological systems is significant in both theoretical and practical point of views. In fact, when immune system works against the non-self-cells, it may take some time (time lag) to interact with the pathogen. Therefore, time delays cannot be ignored in models for immune response. Accordingly, the analysis of dynamical properties of system with time delays is important (see [5, 7-12]). Dengue fever is analyzed in [13], using a system of four nonlinear differential equations with two time delays. In [12], the authors considered the vectorborne epidemic model with time delay. The authors intensively discussed the impact of time delay in the host-tovector transmission term that can destabilize the system. Periodic solutions can also be raised through Hopf bifurcation.

In the existing literature, most of the biological problems are studied through the integer-order mathematical modeling 
by using ordinary, partial, and delay differential equations $[9,10,14]$. In the last few decades, fractional-order models have been incorporated in several areas of science, engineering, applied mathematics, economics, and bioengineering [15-20]. One advantage of the fractional-order differential equation is that they provide a powerful instrument for incorporation of memory and hereditary properties of the systems as opposed to the integer-order models, where such effects are neglected or difficult to incorporate. In addition, when fitting data, the fractional models have one more degree of freedom than the integer-order model (see [21]). Based on these advantages, some authors have developed interesting applications to investigate the dynamics of such fractionalorder models with systems of memory [22-26]. In [5, 22], the authors studied fractional-order cancer immune systems. In [25], a fractional-order model for HIV with nonlinear incidence has been considered and stability for various equilibrium points has also been discussed. The authors in [27] investigated the dynamics of Ebola virus with time delay and fractional order and reported that combination of time delay and fractional order can effectively enrich the dynamics and strengthen the stability condition of the infection model. Analysis and dynamics of Zika transmission have been examined by many researchers (see, e.g., [3, 28, 29]. In [3], a mathematical model for transmission of Zika virus has been proposed with control measures of Zika virus. Stability properties of the Zika infection model have been investigated in [30]. The authors in [31] have compared the Zika infection model with dengue to show effect of the virus on population. The dynamical analysis of the SIS model is studied by considering bifurcation parameters in [32]. The authors [33] have discussed absence and presence of diffusion in the Zika virus disease model. The stability analysis and Hopf bifurcation point for various generalized epidemic models have been discussed in the literature [33-35]. However, the dynamics of fractional order with multiple time-delay models for Zika virus infection has not been yet studied in mathematical epidemiology.

Herein, we demonstrate that a nonlinear fractional-order differential equations model, with multiple time delays, can simulate the dynamics of Zika virus infection much more than the classical epidemic models. The application of fractional derivatives is in several cases justified because they provide a better model than integer-order derivative models do [36, 37]. One important feature of fractional derivatives is that they are nonlocal opposed to the local behaviour of integer derivatives. In this way, the next state of a fractional system depends not only upon its current state but also upon all of its historical states [38-40].

Motivated by the above discussion, in this paper, we investigate the dynamics of Zika virus infection with fractional order and time delays. In Section 2, we formulate the model and study the nonnegativity of the solutions. In Section 3, we investigate the asymptotic stability analysis and Hopf bifurcation properties by taking time-delay parameters as bifurcation parameters. Sufficient conditions are derived to ensure the asymptotic stability and Hopf bifurcation behaviours of the addressed model. Finally, some numerical simulations are provided with various fractional orders and time delays to demonstrate the effectiveness of our theoretical findings in Section 4. We then conclude in Section 5.

Before we start analysis, we provide some useful preliminaries.

1.1. Preliminaries. Herein, we provide some basic definitions and properties of integration and differentiation with fractional-order (free order) $\alpha$ (see [41]).

Definition 1. Let $\alpha \in(0, \infty)$, the operator $I_{a}^{\alpha}$ on $L_{1}[a, b]$ is defined by

$$
I_{a}^{\alpha} f(t)=\frac{1}{\Gamma(\alpha)} \int_{a}^{t}(t-s)^{\alpha-1} f(s) \mathrm{d} s, \quad f \in L_{1}[a, b], t \in[a, b],
$$

which is called the fractional integral (or Riemann-Liouville integral) of order $\alpha$, where $I_{a}^{0}=\mathrm{Id}$ is the identity operator.

Definition 2. Let $\alpha \in[0, \infty)$ and $n=[\alpha]$, where $[x]=\min \{k \in \mathbb{Z}: k \geq x\}$, and the operator ${ }_{\mathrm{RL}} D_{a}^{\alpha}$ is defined for $f \in L_{1}[a, b]$ by

$$
{ }_{\mathrm{RL}} D_{a}^{\alpha} f(t)=\frac{1}{\Gamma(n-\alpha)}\left(\frac{\mathrm{d}}{\mathrm{d} t}\right)^{n} \int_{a}^{t}(t-s)^{n-\alpha-1} f(s) \mathrm{d} s,
$$

which is called the Riemann-Liouville fractional derivative of order $\alpha$.

Definition 3. Let $\alpha \in[0, \infty)$ and $f$ is such that $I_{a}^{n-\alpha} f^{(n)}$ exists, where $n=[\alpha], f \in A^{n}[a, b]$ (the set of all function $f:[a, b] \longrightarrow \mathbb{R}$ provided that $f^{(n-1)}$ be absolutely continuous), then we define the operator ${ }_{C} D_{a}^{\alpha}$ by

$$
{ }_{C} D_{a}^{\alpha} f(t)=\frac{1}{\Gamma(n-\alpha)} \int_{a}^{t}(t-s)^{n-\alpha-1} f^{(n)}(s) \mathrm{d} s,
$$

which exists for almost everywhere $x \in[a, b]$. The operator ${ }_{C} D_{a}^{\alpha} f(t)$ is called the Caputo fractional derivative of order $\alpha$. In particular, when $0<\alpha \leq 1$, we have

$$
{ }_{C} D_{a}^{\alpha} f(t)=\frac{1}{\Gamma(1-\alpha)} \int_{a}^{t} \frac{f^{\prime}(s)}{(t-s)^{\alpha}} \mathrm{d} s .
$$

Remark 1. Let $\beta, \gamma \in \mathbb{R}_{+}$and $\alpha \in(0,1)$. Then,

(i) If $I_{a}^{\beta}: L_{1} \longrightarrow L_{1}$ and if $f(t) \in L_{1}$, then $I_{a}^{\beta} I_{a}^{\gamma} f^{a}(t)=I_{a}^{\beta+\gamma} f(t)$

(ii) $\lim _{\beta \longrightarrow n} I_{a}^{\beta} f(x)=I_{a}^{n} f(t) \quad$ uniformly on $[a, b]$, $n=1,2,3, \ldots$, where $I_{a}^{1} f(t)=\int_{a}^{t} f(s) \mathrm{d} s$

(iii) $\lim _{\beta \longrightarrow 0} I_{a}^{\beta} f(t)=f(t)$ weakly

(iv) If $f(t)$ is absolutely continuous on $[a, b]$, then $\lim _{\alpha \longrightarrow 1} D_{a}^{\alpha} f(t)=\mathrm{d} f(t) / \mathrm{d} t$

(v) Thus, $\quad D_{a}^{\alpha} f(t)=(\mathrm{d} / \mathrm{d} t) I_{a}^{1-\alpha} f(t) \quad$ (RiemannLiouville sense) and $D^{\alpha} f(t)=I_{a}^{1-\alpha}(\mathrm{d} / \mathrm{d} t) f(t)$ (Caputo sense) 
Remark 2. We notice that the fractional derivatives involve an integration and are nonlocal operators, which can be used for modeling systems with memory.

We should mention here that Caputo's definition of fractional derivative is a modification of the RiemannLiouville definition and has the advantage of dealing with initial value problems in a proper way.

\section{Model Formulation}

The literature reveals that most mathematical modeling of biological systems with memory is based either on delay differential equations (DDEs) with integer-order or fractional-order differential equations without a delay. However, fractional-order calculus is more suitable, than integer-order ones, in modeling biological systems with intrinsic memory and long-range interactions such as epidemic evolution systems [42]. Modeling of such systems by fractional-order differential equations has more advantages than classical integer-order mathematical modeling, in which the effects of memory or long-range interactions are neglected. Indeed, memory effects play an essential role in the spreading of diseases. Including memory effects in the susceptible-infected-recovered (SIR) epidemic models seems very appropriate for such an investigation (see Remark 2). Herein, we investigate the impact of combining both time delays and fractional order in an epidemic model for Zika virus infection.

The underlying model is governed by a system of fractional-order differential equations with multiple time delays for Zika virus infection. The model includes the dynamics of susceptible individuals, $H_{S}(t)$, with Zika symptoms and infected portion, $H_{I}(t)$, and recovered portion, $H_{R}(t)$, individuals recovered from Zika, the susceptible mosquitoes, $M_{S}(t)$, in infected mosquitoes, $M_{I}(t)$. Thus, the total human population $N_{H}(t)=H_{s}(t)+$ $H_{I}(t)+H_{R}(t)$. The overall vector (mosquito) population, at time $t$, is $N_{m}(t)=M_{s}(t)+M_{I}(t)$. Assume that $\beta_{h}$ is the transmission rate from humans to mosquitoes. $\beta_{m}$ is the transmission rate of Zika from the vector (mosquitoes) to humans. Natural death rate of host is denoted by $d_{h}$. The recruitment rate into susceptible population is denoted by $\lambda_{h}$. Natural death rate of vector is denoted by $d_{m} . \eta$ is the recovery rate from treatment. $\lambda_{m}$ is the recruitment rate into susceptible mosquito population. Also, $\gamma$ is the average infectious period for humans. We use time delays in the model to consider the latency of the infection in a vector and the latency of the infection in an infected host. In our model, we consider time-delay $\tau_{1}$ to represent the transferring of the infection from infected mosquitoes into suspected humans. The incubation period (time delay) $\tau_{2}$ is incorporated to represent the time required for an individual/susceptible to become infectious, after becoming infected. $\tau_{3}$ is the incubation period of susceptible mosquitoes to become infectious (see Figure 1). The memory of the earlier times, which are represented by time lags, could have less effect on the present situation, as compared to more recent times. However, it is expected that long-range memory, represented by fractional order, effects decay in time more slowly than an exponential decay but can typically behave like a power-law damping function. The model then takes the following form:

$$
\begin{aligned}
D^{\alpha} H_{S}(t)= & \lambda_{h}-\beta_{h} H_{S}\left(t-\tau_{1}\right) M_{I}\left(t-\tau_{1}\right) \\
& -\beta_{h} H_{S}\left(t-\tau_{2}\right) H_{I}\left(t-\tau_{2}\right)-d_{h} H_{S} \\
D^{\alpha} H_{I}(t)= & \beta_{h} H_{S}\left(t-\tau_{1}\right) M_{I}\left(t-\tau_{1}\right) \\
& +\beta_{h} H_{S}\left(t-\tau_{2}\right) H_{I}\left(t-\tau_{2}\right)-d_{h} H_{I}-\gamma H_{I}, \\
D^{\alpha} H_{R}(t)= & \gamma H_{I}-d_{h} H_{R}+\eta H_{I} \\
D^{\alpha} M_{S}(t)= & \lambda_{m}-\beta_{m} M_{S}\left(t-\tau_{3}\right) H_{I}\left(t-\tau_{3}\right)-d_{m} M_{s}, \\
D^{\alpha} M_{I}(t)= & \beta_{m} M_{S}\left(t-\tau_{3}\right) H_{I}\left(t-\tau_{3}\right)-d_{m} M_{I} .
\end{aligned}
$$

The initial conditions for system (5) should be provided so that $H_{R}(0)=H_{R_{0}}, \quad H_{S}(t)=\phi_{1}(t), \quad H_{I}(t)=\phi_{2}(t)$, $M_{S}(t)=\phi_{3}(t)$, and $M_{I}(t)=\phi_{4}(t)$, when $t \in\left[\max \left\{-\tau_{i}\right\}, 0\right]$ for $i=1,2,3$., time lag, $\tau_{i} \geq 0$.

Remark 3. The fractional derivative $\alpha \in(0,1]$ is defined by Caputo sense (4), so that introducing a convolution integral with a power-law memory kernel is useful to describe memory effects in dynamical systems. The decaying rate of the memory kernel (a time correlation function) depends on $\alpha$. A lower value of $\alpha$ corresponds to more slowly decaying time-correlation functions (long memory). Therefore, as $\alpha \longrightarrow 1$, the influence of memory decreases.

2.1. Nonnegative Solution. Since model (5) monitors the dynamics of human populations, therefore, all the parameters are assumed to be nonnegative. Furthermore, it can be shown that all state variables of the model are nonnegative and bounded for all time $t \geq 0$ (see [42]).

Lemma 1. The closed set $\Omega=\left\{\left(H_{s}, H_{I}, H_{R}, M_{S}, M_{I}\right) \in \mathbb{R}_{+}^{5}\right.$ : $\left.H_{S}+H_{I}+H_{R} \leq\left(\lambda_{h} / d_{h}\right), M_{S}+M_{I} \leq\left(\lambda_{m} / d_{m}\right)\right\}$ is positively invariant with respect to model (5).

Proof. In order to prove the nonnegativity of system (5), it is assumed that there exists a $t_{*}>t_{0}$ such that $H_{S}\left(t_{*}\right)=0$ and $H_{S}(t)<0$ for $t \in\left(t_{*}, t_{1}\right]$ where $t_{1}$ is sufficiently close to $t_{*}$. If $H_{S}(t)=0$,

$$
D^{\alpha} H_{S}\left(t_{*}\right)=\lambda_{h}
$$

Thus, one obtains $D^{\alpha} H_{S}(t)>0$ for all $t \in\left[t_{*}, t_{1}\right]$ and $D^{\alpha} H_{S}>\epsilon H_{S}$, where $\epsilon>0$. Hence, one derives

$$
H_{S}(t)>H_{S}\left(t_{*}\right) E_{\alpha}\left(\epsilon\left(t-t_{*}\right)^{\alpha}\right), \quad t \in\left[t_{*}, t_{1}\right] .
$$

Since $H_{S}\left(t_{*}\right)=0$, one gets $H_{S}(t)>0, t \in\left[t_{*}, t_{1}\right]$, which contradicts the assumption. Hence, $H_{S}(t)>0$ for any $t>t_{0}$. In the same manner, we have $H_{I}(t), H_{R}(t)$, $M_{S}(t)$, and $M_{I}(t)$ are nonnegative.

To show that the system is bounded, we add the first three equation of System (5), and we get 


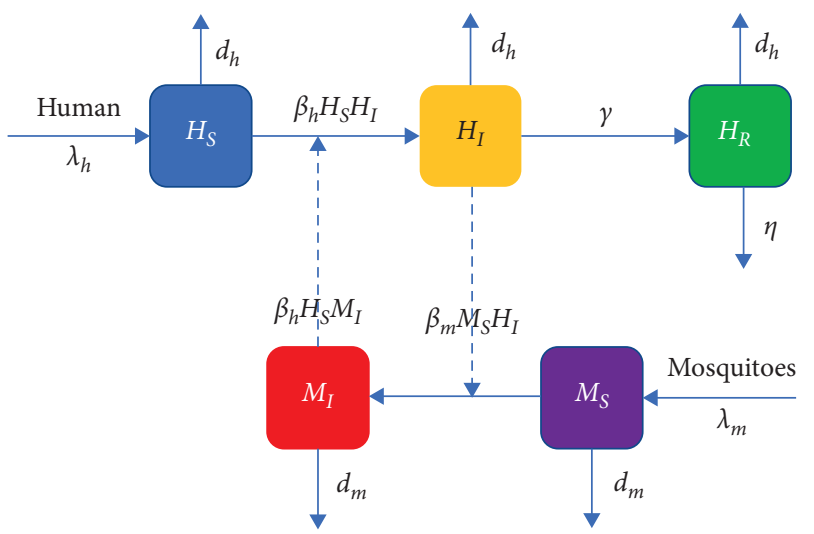

Figure 1: Transmission and dynamics of Zika virus infection between the host (human) and the vector (mosquitoes).

$$
D^{\alpha}\left(H_{S}+H_{I}+H_{R}\right)=\lambda_{h}-d_{h} H_{s}-d_{h} H_{I}-d_{h} H_{R}+\eta H_{I}
$$

We know that all parameters value is positive, and one can obtain

$$
\begin{gathered}
D^{\alpha}\left(H_{S}+H_{I}+H_{R}\right) \leq \lambda_{h}-d_{h}\left(H_{S}+H_{I}+H_{R}\right), \\
D^{\alpha} N_{H} \leq \lambda_{h}-d_{h} N_{H},
\end{gathered}
$$

where $N_{H}=H_{S}(t)+H_{I}(t)+H_{R}(t)$, and solving this equation, we have

$$
N_{H}(t) \leq\left(-\frac{\lambda_{h}}{d_{h}}+N_{H}(0)\right) E_{\alpha}\left(-d_{h} t^{\alpha}\right)+\frac{\lambda_{h}}{d_{h}} .
$$

The solution is given by $N_{H}(t)=N_{H}(0) E_{\alpha, 1}$ $\left(-d_{h} t^{\alpha}\right)+\lambda_{h} t^{\alpha} E_{\alpha, \alpha+1}\left(-d_{h} t^{\alpha}\right)$, where $E_{\alpha, \beta}$ is the Mittag-Leffler function. Considering the fact that Mittag-Leffler function has an asymptotic behaviour,

$$
\begin{aligned}
E_{\alpha, \beta}(z) \sim-\sum_{K=1}^{\omega} \frac{z^{-K}}{\Gamma(\beta-\alpha K)}+\mathcal{O}\left(|z|^{-1-\omega}\right), \\
|z| \longrightarrow \infty, \frac{\alpha \pi}{2}<|\arg (z)| \leq \pi
\end{aligned}
$$

One can observe that $N_{H}(t) \longrightarrow \lambda_{h} / d_{h}$ as $t \longrightarrow \infty$. The proof of the mosquitoes (vector) population is similar to human (host) population, and we obtain $N_{M}(t) \longrightarrow \lambda_{m} / d_{m}$. Therefore, all solutions of the model with initial conditions in $\Omega$ remain bounded in the positively invariant region $\Omega$ for all $t \in[0, \infty)$. The region $\Omega$ is positively invariant with respect to model (5).

The equilibrium points (steady states) are obtained by setting $D^{\alpha} H_{S}=D^{\alpha} H_{I}=D^{\alpha} H_{R}=D^{\alpha} M_{S}=D^{\alpha} M_{I}=0$, in model (5). The model has two equilibrium points: (i) disease-free equilibrium point $E^{0}=\left(H_{S}^{0}, H_{I}^{0}, H_{R}^{0}, M_{I}^{0}, M_{R}^{0}\right)$ $=\left(\left(\lambda_{h} / d_{h}\right), 0,0,\left(\lambda_{m} / d_{m}\right), 0\right)$ and (ii) endemic steady state $E^{*}$, which is

$$
\begin{gathered}
E^{*}\left(\frac{\lambda_{h}}{\beta_{h}\left(\beta_{m} \lambda_{m} H_{I}^{*} / d_{m}\left(\beta_{m} H_{I}^{*}+d_{m}\right)\right)+\beta_{h} H_{I}^{*}+d_{h}},\right. \\
\left.H_{I}^{*}, \frac{H_{I}^{*}(\eta+\gamma)}{d_{h}}, \frac{\lambda_{m}}{\beta_{m} H_{I}^{*}+d_{m}}, \frac{\beta_{m} \lambda_{m} H_{I}^{*}}{d_{m}\left(\beta_{m} H_{I}^{*}+d_{m}\right)}\right) .
\end{gathered}
$$

Here, $H_{I}^{*}$ is the positive root of the following equation:

$$
\begin{aligned}
& \beta_{h}\left(\frac{\lambda_{h}}{\beta_{h}\left(\beta_{m} \lambda_{m} H_{I}^{*} / d_{m}\left(\beta_{m} H_{I}^{*}+d_{m}\right)\right)+\beta_{h} H_{I}^{*}+d_{h}} \frac{\beta_{m} \lambda_{m} H_{I}^{*}}{d_{m}\left(\beta_{m} H_{I}^{*}+d_{m}\right)}\right)+ \\
& \beta_{h}\left(\frac{\lambda_{h}}{\beta_{h}\left(\beta_{m} \lambda_{m} H_{I}^{*} / d_{m}\left(\beta_{m} H_{I}^{*}+d_{m}\right)\right)+\beta_{h} H_{I}^{*}+d_{h}} H_{I}^{*}\right)-d_{h} H_{I}^{*}-\gamma H_{I}^{*}=0 .
\end{aligned}
$$




\section{Stability and Bifurcation Analysis}

To study the stability of model (5), suppose $E^{*}\left(H_{S}^{*}, H_{I}^{*}, H_{R}^{*}, M_{S}^{*}, M_{I}^{*}\right)$ is the steady state of the linearized system:

$$
\begin{aligned}
& D^{\alpha} H_{S}(t)=-\beta_{h} H_{S}^{*} M_{I}\left(t-\tau_{1}\right)-\beta_{h} M_{I}^{*} H_{S}\left(t-\tau_{1}\right)-\beta_{h} H_{S}^{*} H_{I}\left(t-\tau_{2}\right)-\beta_{h} H_{I}^{*} H_{S}\left(t-\tau_{2}\right)-d_{h} H_{S}, \\
& D^{\alpha} H_{I}(t)=\beta_{h} H_{S}^{*} M_{I}\left(t-\tau_{1}\right)+\beta_{h} M_{I}^{*} H_{S}\left(t-\tau_{1}\right)+\beta_{h} H_{S}^{*} H_{I}\left(t-\tau_{2}\right)+\beta_{h} H_{I}^{*} H_{S}\left(t-\tau_{2}\right)-d_{h} H_{I}-\gamma H_{I}, \\
& D^{\alpha} H_{R}(t)=\gamma H_{I}+\eta H_{I}-d_{h} H_{R}, \\
& D^{\alpha} M_{S}(t)=-\beta_{m} M_{S}^{*} H_{I}\left(t-\tau_{3}\right)-\beta_{m} H_{I}^{*} M_{S}\left(t-\tau_{3}\right)-d_{m} M_{S}, \\
& D^{\alpha} M_{I}(t)=\beta_{m} M_{S}^{*} H_{I}\left(t-\tau_{3}\right)+\beta_{m} H_{I}^{*} M_{S}\left(t-\tau_{3}\right)-d_{m} M_{I} .
\end{aligned}
$$

Taking Laplace transform [43] on both sides of the linearized system (14), we obtain

$$
\begin{aligned}
s^{\alpha} X_{1}(s)= & s^{\alpha-1} \varphi_{1}(0)+\beta_{h} H_{S}^{*} e^{-s \tau_{1}}\left[-X_{5}(s)-\int_{-\tau_{1}}^{0} e^{-s t} \varphi_{5}(t) d t\right] \\
& +\beta_{h} M_{I}^{*} e^{-s \tau_{1}}\left[-X_{1}(s)-\int_{-\tau_{1}}^{0} e^{-s t} \varphi_{1}(t) d t\right]+\beta_{h} H_{S}^{*} e^{-s \tau_{2}}\left[-X_{2}(s)-\int_{-\tau_{2}}^{0} e^{-s t} \varphi_{2}(t) d t\right] \\
& +\beta_{h} H_{I}^{*} e^{-s \tau_{2}}\left[-X_{1}(s)-\int_{-\tau_{2}}^{0} e^{-s t} \varphi_{1}(t) d t\right]-d_{h} X_{1}(s), \\
s^{\alpha} X_{2}(s)= & s^{\alpha-1} \varphi_{2}(0)+\beta_{h} H_{S}^{*} e^{-s \tau_{1}}\left[X_{5}(s)+\int_{-\tau_{1}}^{0} e^{-s t} \varphi_{5}(t) d t\right] \\
& +\beta_{h} M_{I}^{*} e^{-s \tau_{1}}\left[X_{1}(s)+\int_{-\tau_{1}}^{0} e^{-s t} \varphi_{1}(t) d t\right]+\beta_{h} H_{S}^{*} e^{-s \tau_{2}}\left[X_{2}(s)+\int_{-\tau_{2}}^{0} e^{-s t} \varphi_{2}(t) d t\right] \\
& +\beta_{h} H_{I}^{*} e^{-s \tau_{2}}\left[X_{1}(s)+\int_{-\tau_{2}}^{0} e^{-s t} \varphi_{1}(t) d t\right]-d_{h} X_{2}(s)-\gamma X_{2}(s), \\
s^{\alpha} X_{3}(s)= & s^{\alpha-1} \varphi_{3}(0)+\gamma X_{2}(s)+\eta X_{2}(s)-d_{h} X_{3}(s), \\
s^{\alpha} X_{4}(s)= & s^{\alpha-1} \varphi_{4}(0)+\beta_{m} M_{S}^{*} e^{-s \tau_{3}}\left[-X_{2}(s)-\int_{-\tau_{3}}^{0} e^{-s t} \varphi_{2}(t) d t\right] \\
& +\beta_{m} H_{I}^{*} e^{-s \tau_{3}}\left[-X_{4}(s)-\int_{-\tau_{3}}^{0} e^{-s t} \varphi_{4}(t) d t\right]-d_{m} X_{4}(s), \\
& \left.+\beta_{m}^{\alpha-1} \varphi_{5}(0)+\beta_{m} M_{S}^{*} e^{-s \tau_{3}}\left[X_{2}(s)+\int_{-\tau_{3}}^{0} e^{-s t} \varphi_{2}(t) d t\right]+\int_{-\tau_{3}}^{0} e^{-s t} \varphi_{4}(t) d t\right]-d_{m} X_{5}(s), \\
s^{\alpha} X_{5}(s) &
\end{aligned}
$$

where $X_{1}(s), X_{2}(s), X_{3}(s), X_{4}(s)$, and $X_{5}(s)$ are Laplace transforms of $H_{S}, H_{I}, H_{R}, M_{S}$, and $M_{I}$, respectively, with $X_{1}(s)=\mathscr{L}\left\{H_{S}(t)\right\}, X_{2}(s)=\mathscr{L}\left\{H_{I}(t)\right\}, X_{3}(s)=\mathscr{L}\left\{H_{R}(t)\right\}$, $X_{4}(s)=\mathscr{L}\left\{M_{S}(t)\right\}$, and $X_{5}(s)=\mathscr{L}\left\{M_{I}(t)\right\}$. Then, (15) can be written in the following matrix form as

$$
\Delta(s)\left(\begin{array}{l}
X_{1}(s) \\
X_{2}(s) \\
X_{3}(s) \\
X_{4}(s) \\
X_{5}(s)
\end{array}\right)=\left(\begin{array}{l}
k_{1}(s) \\
k_{2}(s) \\
k_{3}(s) \\
k_{4}(s) \\
k_{5}(s)
\end{array}\right),
$$


in which

$$
\begin{aligned}
& \Delta(s)=\left(\begin{array}{ccccc}
s^{\alpha}+a_{1} e^{-s \tau_{1}}+a_{2} e^{-s \tau_{2}}+a_{3} & a_{4} e^{-s \tau_{2}} & 0 & 0 & a_{4} e^{-s \tau_{1}} \\
-a_{1} e^{-s \tau_{1}}-a_{2} e^{-s \tau_{2}} & s^{\alpha}-a_{4} e^{-s \tau_{2}}+a_{5} & 0 & 0 & -a_{4} e^{-s \tau_{1}} \\
0 & a 6 & s^{\alpha}+a_{3} & 0 & 0 \\
0 & a_{7} e^{-s \tau_{3}} & 0 & s^{\alpha}+a_{8} e^{-s \tau_{3}}+a_{9} & 0 \\
0 & -a_{7} e^{-s \tau_{3}} & 0 & -a_{8} e^{-s \tau_{3}} & s^{\alpha}+a_{9}
\end{array}\right), \\
& k_{1}(s)=s^{\alpha-1} \varphi_{1}(0)-\beta_{h} e^{-s \tau_{1}} H_{S}^{*} \int_{-\tau_{1}}^{0} e^{-s t} \varphi_{5}(t) \mathrm{d} t-\beta_{h} e^{-s \tau_{1}} M_{I}^{*} \int_{-\tau_{1}}^{0} e^{-s t} \varphi_{1}(t) \mathrm{d} t \\
& -\beta_{h} e^{-s \tau_{2}} H_{S}^{*} \int_{-\tau_{2}}^{0} e^{-s t} \varphi_{2}(t) \mathrm{d} t-\beta_{h} e^{-s \tau_{2}} H_{I}^{*} \int_{-\tau_{2}}^{0} e^{-s t} \varphi_{1}(t) \mathrm{d} t, \\
& k_{2}(s)=s^{\alpha-1} \varphi_{2}(0)+\beta_{h} e^{-s \tau_{1}} H_{S}^{*} \int_{-\tau_{1}}^{0} e^{-s t} \varphi_{5}(t) \mathrm{d} t+\beta_{h} e^{-s \tau_{1}} M_{I}^{*} \int_{-\tau_{1}}^{0} e^{-s t} \varphi_{1}(t) \mathrm{d} t \\
& +\beta_{h} e^{-s \tau_{2}} H_{S}^{*} \int_{-\tau_{2}}^{0} e^{-s t} \varphi_{2}(t) \mathrm{d} t+\beta_{h} e^{-s \tau_{2}} H_{I}^{*} \int_{-\tau_{2}}^{0} e^{-s t} \varphi_{1}(t) \mathrm{d} t, \\
& k_{3}(s)=s^{\alpha-1} \varphi_{3}(0) \text {, } \\
& k_{4}(s)=s^{\alpha-1} \varphi_{4}(0)-\beta_{m} M_{S}^{*} e^{-s \tau_{3}} \int_{-\tau_{3}}^{0} e^{-s t} \varphi_{2}(t) \mathrm{d} t-\beta_{m} e^{-s \tau_{3}} H_{I}^{*} \int_{-\tau_{3}}^{0} e^{-s \tau_{3}} \varphi_{4}(t) \mathrm{d} t, \\
& k_{5}(s)=s^{\alpha-1} \varphi_{5}(0)+\beta_{m} M_{S}^{*} e^{-s \tau_{3}} \int_{-\tau_{3}}^{0} e^{-s t} \varphi_{2}(t) \mathrm{d} t+\beta_{m} e^{-s \tau_{3}} H_{I}^{*} \int_{-\tau_{3}}^{0} e^{-s \tau_{3}} \varphi_{4}(t) \mathrm{d} t,
\end{aligned}
$$

where $a_{1}=\beta_{h} M_{I}^{*}, a_{2}=\beta_{h} H_{I}^{*}, a_{3}=d_{h}, a_{4}=\beta_{h} H_{S}^{*}, a_{5}=d_{h}+$ $\gamma, a_{6}=-\eta-\gamma, a_{7}=\beta_{m} M_{S}^{*}, a_{8}=\beta_{m} H_{I}^{*}$, and $a_{9}=d_{m}$ and $\Delta(s)$ is considered as the characteristic matrix of system (5) The characteristic polynomial is then

$$
\begin{aligned}
\mathscr{P}(s)= & \mathbb{P}_{1}(s)+\mathbb{P}_{2}(s) e^{-s \tau_{1}}+\mathbb{P}_{3}(s) e^{-s \tau_{2}}+\mathbb{P}_{4}(s) e^{-s \tau_{3}} \\
& +\mathbb{P}_{5}(s) e^{-2 s \tau_{1}}+\mathbb{P}_{6}(s) e^{-2 s \tau_{2}} \\
& +\mathbb{P}_{7}(s) e^{-s\left(\tau_{1}+\tau_{2}\right)}+\mathbb{P}_{8}(s) e^{-s\left(\tau_{2}+\tau_{3}\right)} \\
& +\mathbb{P}_{9}(s) e^{-s\left(\tau_{1}+\tau_{3}\right)}+\mathbb{P}_{10}(s) e^{-2 s \tau_{1}-s \tau_{3}} \\
& +\mathbb{P}_{11}(s) e^{-2 s \tau_{2}-s \tau_{3}}+\mathbb{P}_{12}(s) e^{-s\left(\tau_{1}+\tau_{2}+\tau_{3}\right)}
\end{aligned}
$$

The coefficients $\mathbb{P}_{i}(s), i=1, \ldots, 12$, are estimated by Mathematica and given in Appendix.

Case 1. $\tau_{1}>0, \tau_{2}=0$, and $\tau_{3}=0$.

When $\tau_{1}>0, \tau_{2}=0$, and $\tau_{3}=0$, the characteristic equation (18) becomes

$$
\mathscr{P}_{1}(s)+\mathscr{P}_{2}(s) e^{-s \tau_{1}}+\mathscr{P}_{3}(s) e^{-2 s \tau_{1}}=0,
$$

where

$$
\begin{aligned}
\mathscr{P}_{1}(s) & =\mathbb{P}_{1}(s)+\mathbb{P}_{3}(s)+\mathbb{P}_{4}(s)+\mathbb{P}_{6}(s)+\mathbb{P}_{8}(s)+\mathbb{P}_{11}(s) \\
& =s^{5 \alpha}+D_{1} s^{4 \alpha}+D_{2} s^{3 \alpha}+D_{3} s^{2 \alpha}+D_{4} s^{\alpha}+D_{5}, \\
\mathscr{P}_{2}(s) & =\mathbb{P}_{2}(s)+\mathbb{P}_{7}(s)+\mathbb{P}_{9}(s)+\mathbb{P}_{12}(s) \\
& =G_{1} s^{4 \alpha}+G_{2} s^{3 \alpha}+G_{3} s^{2 \alpha}+G_{4} s^{\alpha}+G_{5}, \\
\mathscr{P}_{3}(s) & =\mathbb{P}_{5}(s)+\mathbb{P}_{10}(s)=H_{1} s^{3 \alpha}+H_{2} s^{2 \alpha}+H_{3} s^{\alpha}+H_{4} .
\end{aligned}
$$

Now, we prove that the characteristic equation (19) has no pure imaginary roots for any $\tau_{1}>0$. Assume that characteristic equation (19) has pure imaginary root, and let it be $s=i \xi=\xi(\cos (\pi / 2)+i \sin (\pi / 2)), \xi>0$. If we multiply $e^{s \tau_{1}}$ on both sides of equation (19), we get

$$
\mathscr{P}_{1}(s) e^{s \tau_{1}}+\mathscr{P}_{2}(s)+\mathscr{P}_{3}(s) e^{-s \tau_{1}}=0 .
$$

Now, we substitute the expression of $s$ into (21) to have

$$
\left(\mathscr{A}_{1}+i \mathscr{B}_{1}\right) e^{s \tau_{1}}+\mathscr{A}_{2}+i \mathscr{B}_{2}+\left(\mathscr{A}_{3}+i \mathscr{B}_{3}\right) e^{-s \tau_{1}}=0 \text {. }
$$

The coefficients $\mathscr{A}_{1}, \mathscr{A}_{2}$, and $\mathscr{A}_{3}$ and $\mathscr{B}_{1}, \mathscr{B}_{2}$, and $\mathscr{B}_{3}$ are real and imaginary parts of $\mathscr{P}_{1}(s), \mathscr{P}_{2}(s)$, and $\mathscr{P}_{3}(s)$, respectively, so that 


$$
\begin{aligned}
& \mathscr{A}_{1}=\xi^{5 \alpha} \cos \frac{5 \alpha \pi}{2}+D_{1} \xi^{4 \alpha} \cos \frac{4 \alpha \pi}{2}+D_{2} \xi^{3 \alpha} \cos \frac{3 \alpha \pi}{2} \\
& +D_{3} \xi^{2 \alpha} \cos \frac{2 \alpha \pi}{2}+D_{4} \xi^{\alpha} \cos \frac{\alpha \pi}{2}+D_{5} \\
& \mathscr{B}_{1}=\xi^{5 \alpha} \sin \frac{5 \alpha \pi}{2}+D_{1} \xi^{4 \alpha} \sin \frac{4 \alpha \pi}{2}+D_{2} \xi^{3 \alpha} \sin \frac{3 \alpha \pi}{2} \\
& +D_{3} \xi^{2 \alpha} \sin \frac{2 \alpha \pi}{2}+D_{4} \xi^{\alpha} \sin \frac{\alpha \pi}{2} \\
& \mathscr{A}_{2}=G_{1} \xi^{4 \alpha} \cos \frac{4 \alpha \pi}{2}+G_{2} \xi^{3 \alpha} \cos \frac{3 \alpha \pi}{2}+G_{3} \xi^{2 \alpha} \cos \frac{2 \alpha \pi}{2} \\
& +G_{4} \xi^{\alpha} \cos \frac{\alpha \pi}{2}+G_{5} \\
& \mathscr{B}_{2}=G_{1} \xi^{4 \alpha} \sin \frac{4 \alpha \pi}{2}+G_{2} \xi^{3 \alpha} \sin \frac{3 \alpha \pi}{2}+G_{3} \xi^{2 \alpha} \sin \frac{2 \alpha \pi}{2} \\
& +G_{4} \xi^{\alpha} \sin \frac{\alpha \pi}{2} \\
& \mathscr{A}_{3}=H_{1} \xi^{3 \alpha} \cos \frac{3 \alpha \pi}{2}+H_{2} \xi^{2 \alpha} \cos \frac{2 \alpha \pi}{2}+H_{3} \xi^{\alpha} \cos \frac{\alpha \pi}{2}+H_{4}, \\
& \mathscr{B}_{3}=H_{1} \xi^{3 \alpha} \sin \frac{3 \alpha \pi}{2}+H_{2} \xi^{2 \alpha} \sin \frac{2 \alpha \pi}{2}+H_{3} \xi^{\alpha} \sin \frac{\alpha \pi}{2} .
\end{aligned}
$$

Separating real and imaginary parts yields

$\mathscr{A}_{1} \cos \xi \tau_{1}-\mathscr{B}_{1} \sin \xi \tau_{1}=-\left(\mathscr{A}_{3} \cos \xi \tau_{1}+\mathscr{B}_{3} \sin \xi \tau_{1}+\mathscr{A}_{2}\right)$, $\mathscr{A}_{1} \sin \xi \tau_{1}-\mathscr{B}_{1} \cos \xi \tau_{1}=-\left(\mathscr{B}_{3} \cos \xi \tau_{1}-\mathscr{A}_{3} \sin \xi \tau_{1}+\mathscr{B}_{2}\right)$.

It follows from (14) that

$$
\begin{aligned}
& \mathscr{A}_{1}^{2}+\mathscr{B}_{1}^{2}-\mathscr{A}_{2}^{2}-\mathscr{B}_{2}^{2}-\mathscr{A}_{3}^{2}-\mathscr{B}_{3}^{2}=2\left[\mathscr { B } _ { 3 } \left(\mathscr{A}_{2} \sin \xi \tau_{1}\right.\right. \\
& \left.\left.+\mathscr{B}_{2} \cos \xi \tau_{1}\right)+\mathscr{A}_{3}\left(\mathscr{A}_{2} \cos \xi \tau_{1}-\mathscr{B}_{2} \sin \xi \tau_{1}\right)\right] .
\end{aligned}
$$

Using the fact that $\cos ^{2} \theta+\sin ^{2} \theta=1$, we have $\sin \xi \tau_{1}=\sqrt{1-\cos ^{2} \xi \tau_{1}}$, and then (25) can be written in the following form:

$$
\begin{aligned}
& {\left[\mathscr{A}_{1}^{2}+\mathscr{B}_{1}^{2}-\mathscr{A}_{2}^{2}-\mathscr{B}_{2}^{2}-\mathscr{A}_{3}^{2}-\mathscr{B}_{3}^{2}-2\left(\mathscr{B}_{2} \mathscr{B}_{3}+\mathscr{A}_{2} \mathscr{A}_{3}\right) \cos \xi \tau_{1}\right]^{2}} \\
& \quad=\left[2 \sqrt{1-\cos ^{2} \xi \tau_{1}}\left(\mathscr{B}_{3} \mathscr{A}_{2}-\mathscr{A}_{3} \mathscr{B}_{2}\right)\right]^{2} .
\end{aligned}
$$

It can be concluded from (26) that

$$
Q_{1} \cos ^{2} \xi \tau+Q_{2} \cos \xi \tau+Q_{3}=0
$$

where

$$
\begin{aligned}
Q_{1}= & 4 \mathscr{A}_{2}^{2} \mathscr{A}_{3}^{2}+4 \mathscr{A}_{3}^{2} \mathscr{B}_{2}^{2}+4 \mathscr{A}_{2}^{2} \mathscr{B}_{3}^{2}+4 \mathscr{B}_{2}^{2} \mathscr{B}_{3}^{2}, \\
Q_{2}= & 4\left(\mathscr{B}_{2} \mathscr{B}_{3}+\mathscr{A}_{2} \mathscr{A}_{3}\right)\left(-\mathscr{A}_{1}^{2}-\mathscr{B}_{1}^{2}+\mathscr{A}_{2}^{2}+\mathscr{B}_{2}^{2}+\mathscr{A}_{3}^{2}+\mathscr{B}_{3}^{2}\right), \\
\mathcal{Q}_{3}= & {\left[\mathscr{A}_{1}^{2}-\left(\mathscr{A}_{2}-\mathscr{B}_{3}\right)^{2}-\left(\mathscr{A}_{3}-\mathscr{B}_{1}+\mathscr{B}_{2}\right)\left(\mathscr{A}_{3}+\mathscr{B}_{2}+\mathscr{B}_{1}\right)\right] } \\
& \cdot\left[\mathscr{A}_{1}^{2}-\left(\mathscr{A}_{2}+\mathscr{B}_{3}\right)^{2}-\left(\mathscr{A}_{3}-\mathscr{B}_{1}-\mathscr{B}_{2}\right)\left(\mathscr{A}_{3}+\mathscr{B}_{1}\right)-\mathscr{B}_{2}\right] .
\end{aligned}
$$

The quadratic equation (27) has roots, so we can obtain the expression of $\cos \xi \tau_{1}$ and denote $\cos \xi \tau_{1}=f_{1}(\xi)$, where $f_{1}(\xi)$ is a function of $\xi$.

Substituting the expression of $\cos \xi \tau_{1}=\sqrt{1-\sin ^{2} \xi \tau_{1}}$ into (27), we can get expression of $\sin \xi \tau_{1}$. Assume that $\sin \xi \tau_{2}=f_{2}(\xi)$. Moreover, we have $f_{1}^{2}(\xi)+f_{2}^{2}(\xi)=1$. Thus, it follows from $\cos \xi \tau_{1}=f_{1}(\xi)$ that

$$
\tau_{1}=\frac{1}{\xi}\left[\arccos \left(f_{1}(\xi)\right)+2 k \pi\right], \quad k=0,1,2, \ldots
$$

We suppose that $f_{1}^{2}(\xi)+f_{2}^{2}(\xi)=1$ has at least one positive root, and thus, the bifurcation point is defined as

$$
\tau_{1}^{*}=\min \left\{\tau_{1}^{(k)}\right\}, \quad k=0,1,2, \ldots
$$

Now, differentiating equation (21) with respect to $\tau_{1}$, we obtain

$$
\begin{array}{r}
\mathscr{P}_{1}^{\prime}(s) e^{s \tau_{1}} \frac{\mathrm{d} s}{\mathrm{~d} \tau_{1}}+\mathscr{P}_{1} e^{s \tau_{1}}\left(\tau_{1} \frac{d s}{\mathrm{~d} \tau_{1}}+s\right)+\mathscr{P}_{2}^{\prime}(s) \frac{\mathrm{d} s}{\mathrm{~d} \tau_{1}} \\
+\mathscr{P}_{3}^{\prime}(s) e^{-s \tau_{1}} \frac{\mathrm{d} s}{\mathrm{~d} \tau_{1}}+\mathscr{P}_{3} e^{-s \tau_{1}}\left(-\tau_{1} \frac{\mathrm{d} s}{\mathrm{~d} \tau_{1}}-s\right)=0
\end{array}
$$

where $\mathscr{P}_{1}^{\prime}(s), \mathscr{P}_{2}^{\prime}(s)$, and $\mathscr{P}_{3}^{\prime}(s)$ are derivatives of $\mathscr{P}_{1}(s), \mathscr{P}_{2}(s)$, and $\mathscr{P}_{3}(s)$, respectively. It follows that

$$
\frac{\mathrm{d} s}{\mathrm{~d} \tau_{1}}=\frac{-s \mathscr{P}_{1}(s) e^{s \tau_{1}}+s \mathscr{P}_{3}(s) e^{-s \tau_{1}}}{\mathscr{P}_{1}^{\prime}(s) e^{s \tau_{1}}+\tau_{1} \mathscr{P}_{1}(s) e^{s \tau_{1}}+\mathscr{P}_{2}^{\prime}(s)+\mathscr{P}_{3}^{\prime}(s) e^{-s \tau_{1}}-\tau_{1} \mathscr{P}_{3}(s) e^{-s \tau_{1}}}=\frac{M(s)}{N(s)}
$$

From (32), by some computation, we deduce that

$$
\left.\operatorname{Re}\left(\frac{\mathrm{d} s}{\mathrm{~d} \tau_{1}}\right)\right|_{\tau_{1}=\tau_{1}^{*}, \xi=\xi_{0}}=\frac{M_{1} N_{1}+M_{2} N_{2}}{N_{1}^{2}+N_{2}^{2}}
$$

where $M_{1}, N_{1}$ and $M_{2}, N_{2}$ are the real and imaginary parts of $M(s), N(s)$. Also, $\xi_{0}$ stands for the critical value and $\tau_{1}^{*}$ denotes the bifurcation point. Here, 


$$
\begin{aligned}
& M_{1}=\mathscr{A}_{1} \xi_{0} \sin \xi_{0} \tau_{1}^{*}+\mathscr{B}_{1} \xi_{0} \cos \xi_{0} \tau_{1}^{*}+\mathscr{A}_{3} \xi_{0} \sin \xi_{0} \tau_{1}^{*}-\mathscr{B}_{3} \xi_{0} \sin \xi_{0} \tau_{1}^{*}, \\
& M_{2}=-\mathscr{A}_{1} \xi_{0} \cos \xi_{0} \tau_{1}^{*}+\mathscr{B}_{1} \xi_{0} \sin \xi_{0} \tau_{1}^{*}+\xi_{0} \mathscr{A}_{3} \cos \xi_{0} \tau_{1}^{*}-\mathscr{B}_{3} \xi_{0} \cos \xi_{0} \tau_{1}^{*} \\
& N_{1}=\mathscr{A}_{1}^{*} \cos \xi_{0} \tau_{1}^{*}-\mathscr{B}_{1}^{*} \sin \xi_{0} \tau_{1}^{*}+\tau_{1}^{*} \mathscr{A}_{1} \cos \xi_{0} \tau_{1}^{*}-\tau_{1}^{*} \mathscr{B}_{1} \sin \xi_{0} \tau_{1}^{*}+\mathscr{A}_{2}^{*}+\mathscr{A}_{3}^{*} \cos \xi_{0} \tau_{1}^{*}+B_{3}^{*} \sin \xi_{0} \tau_{1}^{*} \\
& -\tau_{1}^{*} \mathscr{A}_{3} \cos \xi_{0} \tau_{1}^{*}-\tau_{1}^{*} \mathscr{B}_{3} \sin \xi_{0} \tau_{1}^{*}, \\
& N_{2}=\mathscr{A}_{1}^{*} \sin \xi_{0} \tau_{1}^{*}+\mathscr{B}_{1}^{*} \cos \xi_{0} \tau_{1}^{*}+\tau_{1}^{*} \mathscr{A}_{1} \sin \xi_{0} \tau_{1}^{*}+\tau_{1}^{*} \mathscr{B}_{1} \cos \xi_{0} \tau_{1}^{*}+\mathscr{B}_{2}^{*}-\mathscr{A}_{3}^{*} \sin \xi_{0} \tau_{1}^{*}+B_{3}^{*} \cos \xi_{0} \tau_{1}^{*} \\
& +\tau_{1}^{*} \mathscr{A}_{3} \sin \xi_{0} \tau_{1}^{*}-\tau_{1}^{*} \mathscr{B}_{3} \cos \xi_{0} \tau_{1}^{*} \\
& \mathscr{A}_{1}^{*}=5 \alpha \xi_{0}^{5 \alpha-1} \frac{\cos (5 \alpha-1) \pi}{2}+4 \alpha D_{1} \xi_{0}^{4 \alpha-1} \frac{\cos (4 \alpha-1) \pi}{2}+3 \alpha D_{2} \xi_{0}^{3 \alpha-1} \frac{\cos (3 \alpha-1) \pi}{2} \\
& +2 \alpha D_{3} \xi_{0}^{2 \alpha-1} \frac{\cos (2 \alpha-1) \pi}{2}+\alpha D_{4} \xi_{0}^{\alpha-1} \frac{\cos (\alpha-1) \pi}{2} \\
& \mathscr{B}_{1}^{*}=5 \alpha \xi_{0}^{5 \alpha-1} \frac{\sin (5 \alpha-1) \pi}{2}+4 \alpha D_{1} \xi_{0}^{4 \alpha-1} \frac{\sin (4 \alpha-1) \pi}{2}+3 \alpha D_{2} \xi_{0}^{3 \alpha-1} \frac{\sin (3 \alpha-1) \pi}{2} \\
& +2 \alpha D_{3} \xi_{0}^{2 \alpha-1} \frac{\sin (2 \alpha-1) \pi}{2}+\alpha D_{4} \xi_{0}^{\alpha-1} \frac{\sin (\alpha-1) \pi}{2} \\
& \mathscr{A}_{2}^{*}=4 \alpha G_{1} \xi_{0}^{4 \alpha-1} \frac{\cos (4 \alpha-1) \pi}{2}+3 \alpha G_{2} \xi_{0}^{3 \alpha-1} \frac{\cos (3 \alpha-1) \pi}{2}+2 \alpha G_{3} \xi_{0}^{2 \alpha-1} \frac{\cos (2 \alpha-1) \pi}{2}+\alpha G_{4} \xi_{0}^{\alpha-1} \frac{\cos (\alpha-1) \pi}{2}, \\
& \mathscr{B}_{2}^{*}=4 \alpha G_{1} \xi_{0}^{4 \alpha-1} \frac{\sin (4 \alpha-1) \pi}{2}+3 \alpha G_{2} \xi_{0}^{3 \alpha-1} \frac{\sin (3 \alpha-1) \pi}{2}+2 \alpha G_{3} \xi_{0}^{2 \alpha-1} \frac{\sin (2 \alpha-1) \pi}{2}+\alpha G_{4} \xi_{0}^{\alpha-1} \frac{\sin (\alpha-1) \pi}{2} \\
& \mathscr{A}_{3}^{*}=3 \alpha H_{1} \xi_{0}^{3 \alpha-1} \frac{\cos (3 \alpha-1) \pi}{2}+2 \alpha H_{2} \xi_{0}^{2 \alpha-1} \frac{\cos (2 \alpha-1) \pi}{2}+\alpha H_{3} \xi_{0}^{\alpha-1} \frac{\cos (\alpha-1) \pi}{2} \\
& \mathscr{B}_{3}^{*}=3 \alpha H_{1} \xi_{0}^{3 \alpha-1} \frac{\sin (3 \alpha-1) \pi}{2}+2 \alpha H_{2} \xi_{0}^{2 \alpha-1} \frac{\sin (2 \alpha-1) \pi}{2}+\alpha H_{3} \xi_{0}^{\alpha-1} \frac{\sin (\alpha-1) \pi}{2} .
\end{aligned}
$$

Case 2. $\tau_{1}=0, \tau_{2}>0$, and $\tau_{3}=0$.

When $\tau_{1}=0, \tau_{2}>0, \tau_{3}=0$, the characteristic equation (18) becomes

$$
\mathscr{P}_{4}(s)+\mathscr{P}_{5}(s) e^{-s \tau_{2}}+\mathscr{P}_{6}(s) e^{-2 s \tau_{2}}=0,
$$

where

$$
\begin{aligned}
\mathscr{P}_{4}(s)= & \mathbb{P}_{1}(s)+\mathbb{P}_{2}(s)+\mathbb{P}_{4}(s)+\mathbb{P}_{5}(s)+\mathbb{P}_{9}(s) \\
& +\mathbb{P}_{10}(s)=s^{5 \alpha}+J_{1} s^{4 \alpha}+J_{2} s^{3 \alpha}+J_{3} s^{2 \alpha}+J_{4} s^{\alpha}+J_{5}, \\
\mathscr{P}_{5}(s)= & \mathbb{P}_{3}(s)+\mathbb{P}_{7}(s)+\mathbb{P}_{8}(s)+\mathbb{P}_{12}(s)=L_{1} s^{4 \alpha}+L_{2} s^{3 \alpha} \\
& +L_{3} s^{2 \alpha}+L_{4} s^{\alpha}+L_{5}, \\
\mathscr{P}_{6}(s)= & \mathbb{P}_{6}(s)+\mathbb{P}_{11}(s)=R_{1} s^{3 \alpha}+R_{2} s^{2 \alpha}+R_{3} s^{\alpha}+R_{4} .
\end{aligned}
$$

Now, we prove that the characteristic equation (35) has no pure imaginary roots for any $\tau_{2}>0$. Assume that characteristic equation (35) has pure imaginary root, and let it be $s=i \xi=\xi(\cos (\pi / 2)+i \sin (\pi / 2)), \xi>0$. Now, multiplying $e^{s \tau_{2}}$ on both sides of equation (35), we get

$$
\mathscr{P}_{4}(s) e^{s \tau_{2}}+\mathscr{P}_{5}(s)+\mathscr{P}_{6}(s) e^{-s \tau_{2}}=0 \text {. }
$$

Substitute the expression of $s$ into (37) to have

$$
\left(\mathscr{A}_{4}+i \mathscr{B}_{4}\right) e^{s \tau_{2}}+\mathscr{A}_{5}+i \mathscr{B}_{5}+\left(\mathscr{A}_{6}+i \mathscr{B}_{6}\right) e^{-s \tau_{2}}=0,
$$

where $\mathscr{A}_{4}, \mathscr{A}_{5}, \mathscr{A}_{6}$ and $\mathscr{B}_{4}, \mathscr{B}_{5}, \mathscr{B}_{6}$ are real and imaginary parts of $\mathscr{P}_{4}(s), \mathscr{P}_{5}(s)$, and $\mathscr{P}_{6}(s)$, respectively. Here, 


$$
\begin{aligned}
\mathscr{A}_{4}= & \xi^{5 \alpha} \cos \frac{5 \alpha \pi}{2}+J_{1} \xi^{4 \alpha} \cos \frac{4 \alpha \pi}{2}+J_{2} \xi^{3 \alpha} \cos \frac{3 \alpha \pi}{2} \\
& +J_{3} \xi^{2 \alpha} \cos \frac{2 \alpha \pi}{2}+J_{4} \xi^{\alpha} \cos \frac{\alpha \pi}{2}+J_{5} \\
\mathscr{B}_{4}= & \xi^{5 \alpha} \sin \frac{5 \alpha \pi}{2}+J_{1} \xi^{4 \alpha} \sin \frac{4 \alpha \pi}{2}+J_{2} \xi^{3 \alpha} \sin \frac{3 \alpha \pi}{2} \\
& +J_{3} \xi^{2 \alpha} \sin \frac{2 \alpha \pi}{2}+J_{4} \xi^{\alpha} \sin \frac{\alpha \pi}{2}, \\
& +L_{4} \xi^{\alpha} \cos \frac{\alpha \pi}{2}+L_{5}, \\
\mathscr{A}_{5}= & L_{1} \xi^{4 \alpha} \cos \frac{4 \alpha \pi}{2}+L_{2} \xi^{3 \alpha} \cos \frac{3 \alpha \pi}{2}+L_{3} \xi^{2 \alpha} \cos \frac{2 \alpha \pi}{2} \\
\mathscr{B}_{5}= & L_{1} \xi^{4 \alpha} \sin \frac{4 \alpha \pi}{2}+L_{2} \xi^{3 \alpha} \sin \frac{3 \alpha \pi}{2}+L_{3} \xi^{2 \alpha} \sin \frac{2 \alpha \pi}{2} \\
& +L_{4} \xi^{\alpha} \sin \frac{\alpha \pi}{2}, \\
\mathscr{A}_{6}= & R_{1} \xi^{3 \alpha} \cos \frac{3 \alpha \pi}{2}+R_{2} \xi^{2 \alpha} \cos \frac{2 \alpha \pi}{2}+R_{3} \xi^{\alpha} \cos \frac{\alpha \pi}{2}+R_{4}, \\
\mathscr{B}_{6}= & R_{1} \xi^{3 \alpha} \sin \frac{3 \alpha \pi}{2}+R_{2} \xi^{2 \alpha} \sin \frac{2 \alpha \pi}{2}+R_{3} \xi^{\alpha} \sin \frac{\alpha \pi}{2} .
\end{aligned}
$$

Separating real and imaginary parts yields

$\mathscr{A}_{4} \cos \xi \tau_{2}-\mathscr{B}_{4} \sin \xi_{\tau_{2}}=-\left(\mathscr{A}_{6} \cos \xi \tau_{2}+\mathscr{B}_{6} \sin \xi_{2}+\mathscr{A}_{5}\right)$, $\mathscr{A}_{4} \sin \xi \tau_{2}-\mathscr{B}_{4} \cos \xi \tau_{2}=-\left(\mathscr{B}_{6} \cos \xi \tau_{2}-\mathscr{A}_{6} \sin \xi \tau_{2}+\mathscr{B}_{5}\right)$.

It follows from (40) that

$$
\begin{aligned}
\mathscr{A}_{4}^{2} & +\mathscr{B}_{4}^{2}-\mathscr{A}_{5}^{2}-\mathscr{B}_{5}^{2}-\mathscr{A}_{6}^{2}-\mathscr{B}_{6}^{2}=2\left[\mathscr { B } _ { 6 } \left(\mathscr{A}_{5} \sin \xi \tau_{2}\right.\right. \\
& \left.\left.+\mathscr{B}_{5} \cos \xi \tau_{2}\right)+\mathscr{A}_{6}\left(\mathscr{A}_{5} \cos \xi \tau_{2}-\mathscr{B}_{5} \sin \xi \tau_{2}\right)\right] .
\end{aligned}
$$

We know that $\cos ^{2} \theta+\sin ^{2} \theta=1$; by using it, we have $\sin \xi \tau_{2}=\sqrt{1-\cos ^{2} \xi \tau_{2}}$, and then (41) can be written in the following form:

$$
\begin{aligned}
& {\left[\mathscr{A}_{4}^{2}+\mathscr{B}_{4}^{2}-\mathscr{A}_{5}^{2}-\mathscr{B}_{5}^{2}-\mathscr{A}_{6}^{2}-\mathscr{B}_{6}^{2}\right.} \\
& \left.\quad-2\left(\mathscr{B}_{5} \mathscr{B}_{6}+\mathscr{A}_{5} \mathscr{A}_{6}\right) \cos \xi \tau_{2}\right]^{2} \\
& =\left[2 \sqrt{1-\cos ^{2} \xi \tau_{2}}\left(\mathscr{B}_{6} \mathscr{A}_{5}-\mathscr{A}_{6} \mathscr{B}_{5}\right)\right]^{2} .
\end{aligned}
$$

It can be concluded from (42) that

$$
\widehat{Q}_{4} \cos ^{2} \xi \tau+Q_{5} \cos \xi \tau+Q_{6}=0
$$

where

$$
\begin{aligned}
Q_{4}= & 4 \mathscr{A}_{5}^{2} \mathscr{A}_{6}^{2}+4 \mathscr{A}_{6}^{2} \mathscr{B}_{5}^{2}+4 \mathscr{A}_{5}^{2} \mathscr{B}_{6}^{2}+4 \mathscr{B}_{5}^{2} \mathscr{B}_{6}^{2}, \\
\widehat{Q}_{5}= & 4\left(\mathscr{B}_{5} \mathscr{B}_{6}+\mathscr{A}_{5} \mathscr{A}_{6}\right)\left(-\mathscr{A}_{4}^{2}-\mathscr{B}_{4}^{2}+\mathscr{A}_{5}^{2}+\mathscr{B}_{5}^{2}+\mathscr{A}_{6}^{2}+\mathscr{B}_{6}^{2}\right), \\
Q_{6}= & {\left[\mathscr{A}_{4}^{2}-\left(\mathscr{A}_{5}-\mathscr{B}_{6}\right)^{2}-\left(\mathscr{A}_{6}-\mathscr{B}_{4}+\mathscr{B}_{5}\right)\left(\mathscr{A}_{6}+\mathscr{B}_{5}+\mathscr{B}_{4}\right)\right] } \\
& \cdot\left[\mathscr{A}_{4}^{2}-\left(\mathscr{A}_{5}+\mathscr{B}_{6}\right)^{2}-\left(\mathscr{A}_{6}-\mathscr{B}_{4}-\mathscr{B}_{5}\right)\left(\mathscr{A}_{6}+\mathscr{B}_{4}\right)-\mathscr{B}_{5}\right] .
\end{aligned}
$$

As we know, the quadratic equation (43) has roots, we can obtain the expression of $\cos \xi \tau_{2}$ and denote $\cos \xi \tau_{2}=f_{1}(\xi)$, where $f_{1}(\xi)$ is a function of $\xi$.

Substituting the expression of $\cos \xi \tau_{2}=\sqrt{1-\sin ^{2} \xi \tau_{2}}$ into (43), we can get expression of $\sin \xi \tau_{2}$. Let us denote $\sin \xi \tau_{2}=f_{2}(\xi)$, where $f_{2}(\xi)$ is a function with respect to $\xi$. Moreover, $f_{1}^{2}(\xi)+f_{2}^{2}(\xi)=1$. Thus, it follows from $\cos \xi \tau_{2}=$ $f_{1}(\xi)$ that

$$
\tau_{2}=\frac{1}{\xi}\left[\arccos \left(f_{1}(\xi)\right)+2 k \pi\right], \quad k=0,1,2, \ldots
$$

Clearly, $f_{1}^{2}(\xi)+f_{2}^{2}(\xi)=1$ has at least one positive root. The bifurcation point is defined as

$$
\tau_{2}^{*}=\min \left\{\tau_{2}^{(k)}\right\}, \quad k=0,1,2, \ldots
$$

We obtain the transversality condition of the occurrence for Hopf bifurcation at $\tau_{2}=\tau_{2}^{*}$.

Differentiating equation (37) with respect to $\tau_{2}$ yields

$$
\begin{gathered}
\mathscr{P}_{4}^{\prime}(s) e^{s \tau_{2}} \frac{\mathrm{d} s}{\mathrm{~d} \tau_{2}}+\mathscr{P}_{4}(s) e^{s \tau_{2}}\left(\tau_{2} \frac{\mathrm{d} s}{\mathrm{~d} \tau_{2}}+s\right)+\mathscr{P}_{5}^{\prime}(s) \frac{\mathrm{d} s}{\mathrm{~d} \tau_{2}} \\
+\mathscr{P}_{6}^{\prime}(s) e^{-s \tau_{2}} \frac{\mathrm{d} s}{\mathrm{~d} \tau_{2}}+\mathscr{P}_{6}(s) e^{-s \tau_{2}}\left(-\tau_{2} \frac{\mathrm{d} s}{\mathrm{~d} \tau_{2}}-s\right)=0,
\end{gathered}
$$

where $\mathscr{P}_{4}^{\prime}(s), \mathscr{P}_{5}^{\prime}(s)$, and $\mathscr{P}_{6}^{\prime}(s)$ are derivatives of $\mathscr{P}_{4}(s), \mathscr{P}_{5}(s)$, and $\mathscr{P}_{6}(s)$, respectively. It follows that

$$
\frac{\mathrm{d} s}{\mathrm{~d} \tau_{2}}=\frac{-s \mathscr{P}_{4}(s) e^{s \tau_{2}}+s \mathscr{P}_{6}(s) e^{-s \tau_{2}}}{\mathscr{P}_{4}^{\prime}(s) e^{s \tau_{2}}+\tau_{2} \mathscr{P}_{4}(s) e^{s \tau_{2}}+\mathscr{P}_{5}^{\prime}(s)+\mathscr{P}_{6}^{\prime}(s) e^{-s \tau_{2}}-\tau_{2} \mathscr{P}_{6}(s) e^{-s \tau_{1}}}=\frac{\mathscr{M}(s)}{\mathscr{N}(s)}
$$

From (48), by some computation, we deduce that

$$
\left.\operatorname{Re}\left(\frac{\mathrm{d} s}{\mathrm{~d} \tau_{2}}\right)\right|_{\tau_{2}=\tau_{2}^{*}, \xi=\xi_{0}}=\frac{\mathscr{M}_{1} \mathcal{N}_{1}+\mathscr{M}_{2} \mathcal{N}_{2}}{\mathcal{N}_{1}^{2}+\mathscr{N}_{2}^{2}}
$$

where $\mathscr{M}_{1}, \mathcal{N}_{1}$ and $\mathscr{M}_{2}, \mathcal{N}_{2}$ are the real and imaginary parts of $\mathscr{M}(s), \mathscr{N}(s)$. Also $\xi_{0}$ stands for the critical value and $\tau_{2}^{*}$ denotes bifurcation point. Here, 


$$
\begin{aligned}
& \mathscr{M}_{1}=\mathscr{A}_{4} \xi_{0} \sin \xi_{0} \tau_{2}^{*}+\mathscr{B}_{4} \xi_{0} \cos \xi_{0} \tau_{2}^{*}+\mathscr{A}_{6} \xi_{0} \sin \xi_{0} \tau_{2}^{*}-\mathscr{A}_{6} \xi_{0} \sin \xi_{0} \tau_{2}^{*}, \\
& \mathscr{M}_{2}=-\mathscr{A}_{4} \xi_{0} \cos \xi_{0} \tau_{2}^{*}+\mathscr{B}_{4} \xi_{0} \sin \xi_{0} \tau_{2}^{*}+\xi_{0} \mathscr{A}_{6} \cos \xi_{0} \tau_{2}^{*}-\mathscr{B}_{6} \xi_{0} \cos \xi_{0} \tau_{2}^{*} \\
& \mathscr{N}_{1}=\mathscr{A}_{4}^{*} \cos \xi_{0} \tau_{2}^{*}-\mathscr{B}_{2}^{*} \sin \xi_{0} \tau_{2}^{*}+\tau_{2}^{*} \mathscr{A}_{4} \cos \xi_{0} \tau_{2}^{*}-\tau_{2}^{*} \mathscr{B}_{4} \sin \xi_{0} \tau_{2}^{*}+\mathscr{A}_{5}^{*}+\mathscr{A}_{6}^{*} \cos \xi_{0} \tau_{1}^{*}+\mathscr{B}_{6}^{*} \sin \xi_{0} \tau_{2}^{*} \\
& -\tau_{2}^{*} \mathscr{A}_{6} \cos \xi_{0} \tau_{2}^{*}-\tau_{2}^{*} \mathscr{B}_{6} \sin \xi_{0} \tau_{2}^{*} \text {, } \\
& \mathcal{N}_{2}=\mathscr{A}_{4}^{*} \sin \xi_{0} \tau_{2}^{*}+\mathscr{B}_{4}^{*} \cos \xi_{0} \tau_{2}^{*}+\tau_{2}^{*} \mathscr{A}_{4} \sin \xi_{0} \tau_{2}^{*}+\tau_{2}^{*} \mathscr{B}_{4} \cos \xi_{0} \tau_{2}^{*}+\mathscr{B}_{5}^{*}-\mathscr{A}_{6}^{*} \sin \xi_{0} \tau_{2}^{*}+\mathscr{B}_{6}^{*} \cos \xi_{0} \tau_{2}^{*} \\
& +\tau_{2}^{*} \mathscr{A}_{6} \sin \xi_{0} \tau_{2}^{*}-\tau_{2}^{*} \mathscr{B}_{6} \cos \xi_{0} \tau_{2}^{*} \\
& \mathscr{A}_{4}^{*}=5 \alpha \xi_{0}^{5 \alpha-1} \frac{\cos (5 \alpha-1) \pi}{2}+4 \alpha J_{1} \xi_{0}^{4 \alpha-1} \frac{\cos (4 \alpha-1) \pi}{2}+3 \alpha J_{2} \xi_{0}^{3 \alpha-1} \frac{\cos (3 \alpha-1) \pi}{2} \\
& +2 \alpha J_{3} \xi_{0}^{2 \alpha-1} \frac{\cos (2 \alpha-1) \pi}{2}+\alpha J_{4} \xi_{0}^{\alpha-1} \frac{\cos (\alpha-1) \pi}{2} \\
& \mathscr{B}_{4}^{*}=5 \alpha \xi_{0}^{5 \alpha-1} \frac{\sin (5 \alpha-1) \pi}{2}+4 \alpha J_{1} \xi_{0}^{4 \alpha-1} \frac{\sin (4 \alpha-1) \pi}{2}+3 \alpha J_{2} \xi_{0}^{3 \alpha-1} \frac{\sin (3 \alpha-1) \pi}{2} \\
& +2 \alpha J_{3} \xi_{0}^{2 \alpha-1} \frac{\sin (2 \alpha-1) \pi}{2}+\alpha J_{4} \xi_{0}^{\alpha-1} \frac{\sin (\alpha-1) \pi}{2}, \\
& \mathscr{A}_{5}^{*}=4 \alpha L_{1} \xi_{0}^{4 \alpha-1} \frac{\cos (4 \alpha-1) \pi}{2}+3 \alpha L_{2} \xi_{0}^{3 \alpha-1} \frac{\cos (3 \alpha-1) \pi}{2}+2 \alpha L_{3} \xi_{0}^{2 \alpha-1} \frac{\cos (2 \alpha-1) \pi}{2} \\
& +\alpha L_{4} \xi_{0}^{\alpha-1} \frac{\cos (\alpha-1) \pi}{2} \\
& \mathscr{B}_{5}^{*}=4 \alpha L_{1} \xi_{0}^{4 \alpha-1} \frac{\sin (4 \alpha-1) \pi}{2}+3 \alpha L_{2} \xi_{0}^{3 \alpha-1} \frac{\sin (3 \alpha-1) \pi}{2}+2 \alpha L_{3} \xi_{0}^{2 \alpha-1} \frac{\sin (2 \alpha-1) \pi}{2} \\
& +\alpha L_{4} \xi_{0}^{\alpha-1} \frac{\sin (\alpha-1) \pi}{2} \\
& \mathscr{A}_{6}^{*}=3 \alpha R_{1} \xi_{0}^{3 \alpha-1} \frac{\cos (3 \alpha-1) \pi}{2}+2 \alpha R_{2} \xi_{0}^{2 \alpha-1} \frac{\cos (2 \alpha-1) \pi}{2}+\alpha R_{3} \xi_{0}^{\alpha-1} \frac{\cos (\alpha-1) \pi}{2} \\
& \mathscr{B}_{6}^{*}=3 \alpha R_{1} \xi_{0}^{3 \alpha-1} \frac{\sin (3 \alpha-1) \pi}{2}+2 \alpha R_{2} \xi_{0}^{2 \alpha-1} \frac{\sin (2 \alpha-1) \pi}{2}+\alpha R_{3} \xi_{0}^{\alpha-1} \frac{\sin (\alpha-1) \pi}{2} .
\end{aligned}
$$

Case 3. $\tau_{1}=0, \tau_{2}=0$, and $\tau_{3}>0$.

When $\tau_{1}=0, \tau_{2}=0$, and $\tau_{3}>0$, the characteristic equation (18) becomes

$$
\mathscr{P}_{7}(s)+\mathscr{P}_{8}(s) e^{-s \tau_{3}}=0,
$$

where

$$
\begin{aligned}
\mathscr{P}_{7}(s) & =\mathbb{P}_{1}(s)+\mathbb{P}_{2}(s)+\mathbb{P}_{3}(s)+\mathbb{P}_{5}(s)+\mathbb{P}_{6}(s)+\mathbb{P}_{7}(s) \\
& =s^{5 \alpha}+U_{1} s^{4 \alpha}+U_{2} s^{3 \alpha}+U_{3} s^{2 \alpha}+U_{4} s^{\alpha}+U_{5} \\
\mathscr{P}_{8}(s) & =\mathbb{P}_{4}(s)+\mathbb{P}_{8}(s)+\mathbb{P}_{9}(s)+\mathbb{P}_{10}(s)+\mathbb{P}_{11}(s)+\mathbb{P}_{12}(s) \\
& =V_{1} s^{4 \alpha}+V_{2} s^{3 \alpha}+V_{3} s^{2 \alpha}+V_{4} s^{\alpha}+V_{5} .
\end{aligned}
$$

Again, we prove that the characteristic equation (51) has no pure imaginary roots for any $\tau_{2}>0$. Here, we assume that characteristic equation (51) has pure imaginary root, let it be $s=i \xi=\xi(\cos (\pi / 2)+i \sin (\pi / 2)), \xi>0$. Now, we substitute the expression of $s$ into (51), and we have

$$
\mathscr{A}_{7}+i \mathscr{B}_{7}+\left(\mathscr{A}_{8}+i \mathscr{B}_{8}\right) e^{-s \tau_{3}}=0 \text {, }
$$

where $\mathscr{A}_{7}, \mathscr{A}_{8}$ and $\mathscr{B}_{7}, \mathscr{B}_{8}$ are real and imaginary parts of $\mathscr{P}_{7}(s)$ and $\mathscr{P}_{8}(s)$, respectively. Here, 


$$
\begin{aligned}
\mathscr{A}_{7}= & \xi^{5 \alpha} \cos \frac{5 \alpha \pi}{2}+U_{1} \xi^{4 \alpha} \cos \frac{4 \alpha \pi}{2}+U_{2} \xi^{3 \alpha} \cos \frac{3 \alpha \pi}{2} \\
& +U_{3} \xi^{2 \alpha} \cos \frac{2 \alpha \pi}{2}+U_{4} \xi^{\alpha} \cos \frac{\alpha \pi}{2}+U_{5}, \\
\mathscr{B}_{7}= & \xi^{5 \alpha} \sin \frac{5 \alpha \pi}{2}+U_{1} \xi^{4 \alpha} \sin \frac{4 \alpha \pi}{2}+U_{2} \xi^{3 \alpha} \sin \frac{3 \alpha \pi}{2} \\
& +U_{3} \xi^{2 \alpha} \sin \frac{2 \alpha \pi}{2}+U_{4} \xi^{\alpha} \sin \frac{\alpha \pi}{2}, \\
\mathscr{A}_{5}= & V_{1} \xi^{4 \alpha} \cos \frac{4 \alpha \pi}{2}+V_{2} \xi^{3 \alpha} \cos \frac{3 \alpha \pi}{2}+V_{3} \xi^{2 \alpha} \cos \frac{2 \alpha \pi}{2} \\
& +V_{4} \xi^{\alpha} \cos \frac{\alpha \pi}{2}+V_{5}, \\
\mathscr{B}_{5}= & V_{1} \xi^{4 \alpha} \sin \frac{4 \alpha \pi}{2}+V_{2} \xi^{3 \alpha} \sin \frac{3 \alpha \pi}{2}+V_{3} \xi^{2 \alpha} \sin \frac{2 \alpha \pi}{2} \\
& +V_{4} \xi^{\alpha} \sin \frac{\alpha \pi}{2} .
\end{aligned}
$$

Separation of real and imaginary parts yields

$$
\begin{aligned}
\mathscr{A}_{8} \cos \xi \tau_{3}+\mathscr{B}_{8} \sin \xi \tau_{3} & =-\mathscr{A}_{7}, \\
-\mathscr{A}_{8} \sin \xi \tau_{3}+\mathscr{B}_{8} \cos \xi \tau_{3} & =-\mathscr{B}_{7} .
\end{aligned}
$$

From (14), we have

$$
\begin{aligned}
& \cos \xi \tau_{3}=\frac{-\mathscr{A}_{7} \mathscr{A}_{8}-\mathscr{B}_{7} \mathscr{B}_{8}}{\mathscr{A}_{8}^{2}+\mathscr{B}_{8}^{2}}=\bar{f}_{1}(\xi), \\
& \sin \xi \tau_{3}=\frac{\mathscr{B}_{7} \mathscr{A}_{8}-\mathscr{A}_{7} \mathscr{B}_{8}}{\mathscr{A}_{8}^{2}+\mathscr{B}_{8}^{2}}=\bar{f}_{2}(\xi) .
\end{aligned}
$$

It is clear that $\cos ^{2} \theta+\sin ^{2} \theta=1$; from (56),

$$
\left(\bar{f}_{1}(\xi)\right)^{2}+\left(\bar{f}_{2}(\xi)\right)^{2}=1
$$

Hence, it follows from $\cos \xi \tau_{3}=\bar{f}_{1}(\xi)$ that

$$
\tau_{3}=\frac{1}{\xi}\left[\arccos \left(\bar{f}_{1}(\xi)\right)+2 k \pi\right], \quad k=0,1,2, \ldots
$$

We suppose that (57) have at least one positive root. The bifurcation point is defined as

$$
\tau_{3}^{*}=\min \left\{\tau_{3}^{(k)}\right\}, \quad k=0,1,2, \ldots
$$

We obtain the transversality condition of the occurrence for Hopf bifurcation at $\tau_{3}=\tau_{3}^{*}$.

Now, differentiating equation (51) with respect to $\tau_{3}$, we obtain

$$
\mathscr{P}_{7}^{\prime}(s) \frac{\mathrm{d} s}{\mathrm{~d} \tau_{3}}+\mathscr{P}_{8}^{\prime}(s) e^{-s \tau_{3}} \frac{\mathrm{d} s}{\mathrm{~d} \tau_{3}}+\mathscr{P}_{8}(s) e^{-s \tau_{3}}\left(-\tau_{3} \frac{\mathrm{d} s}{\mathrm{~d} \tau_{3}}-s\right)=0,
$$

where $\mathscr{P}_{7}^{\prime}(s)$ and $\mathscr{P}_{8}^{\prime}(s)$ are derivatives of $\mathscr{P}_{7}(s)$ and $\mathscr{P}_{8}(s)$, respectively. It follows that

$$
\frac{\mathrm{d} s}{\mathrm{~d} \tau_{3}}=\frac{-s \mathscr{P}_{8}(s) e^{s \tau_{3}}}{\mathscr{P}_{7}^{\prime}(s)+\mathscr{P}_{8}^{\prime}(s) e^{-s \tau_{3}}-\tau_{3} \mathscr{P}_{8}(s) e^{-s \tau_{3}}}=\frac{\mathscr{M}(s)}{\mathscr{N}(s)} .
$$

From (61), by some computation, we deduce that

$$
\left.\operatorname{Re}\left(\frac{\mathrm{d} s}{\mathrm{~d} \tau_{3}}\right)\right|_{\tau_{3}=\tau_{3}^{*}, \xi=\xi_{0}}=\frac{\mathscr{M}_{1} \mathcal{N}_{1}+\mathscr{M}_{2} \mathcal{N}_{2}}{\mathcal{N}_{1}^{2}+\mathscr{N}_{2}^{2}}
$$

where $\mathscr{M}_{1}, \mathcal{N}_{1}$ and $\mathscr{M}_{2}, \mathcal{N}_{2}$ are the real and imaginary parts of $\mathscr{M}(s), \mathscr{N}(s)$. Also $\xi_{0}$ stands for the critical value and $\tau_{3}^{*}$ denotes bifurcation point. Here,

$$
\begin{aligned}
\mathscr{M}_{1}= & \mathscr{A}_{8} \xi_{0} \sin \xi_{0} \tau_{3}^{*}-\mathscr{B}_{8} \xi_{0} \cos \xi_{0} \tau_{3}^{*}, \\
\mathscr{M}_{2}= & \mathscr{A}_{8} \xi_{0} \cos \xi_{0} \tau_{3}^{*}+\mathscr{B}_{8} \xi_{0} \sin \xi_{0} \tau_{3}^{*}, \\
\mathscr{N}_{1}= & \mathscr{A}_{7}^{*}+\mathscr{A}_{8}^{*} \cos \xi_{0} \tau_{3}^{*}+\mathscr{B}_{8}^{*} \sin \xi_{0} \tau_{3}^{*}-\tau_{3}^{*}\left[\mathscr{A}_{8} \cos \xi_{0} \tau_{3}^{*}+\mathscr{B}_{8} \sin \xi_{0} \tau_{3}^{*}\right] \\
\mathscr{N}_{2}= & \mathscr{B}_{7}^{*}+\mathscr{B}_{8}^{*} \cos \xi_{0} \tau_{3}^{*}-\mathscr{A}_{8}^{*} \sin \xi_{0} \tau_{3}^{*}-\tau_{3}^{*}\left[\mathscr{B}_{8} \cos \xi_{0} \tau_{3}^{*}-\mathscr{A}_{8} \sin \xi_{0} \tau_{3}^{*}\right] \\
\mathscr{A}_{7}^{*}= & 5 \alpha \xi_{0}^{5 \alpha-1} \frac{\cos (5 \alpha-1) \pi}{2}+4 \alpha U_{1} \xi_{0}^{4 \alpha-1} \frac{\cos (4 \alpha-1) \pi}{2}+3 \alpha U_{2} \xi_{0}^{3 \alpha-1} \frac{\cos (3 \alpha-1) \pi}{2} \\
& +2 \alpha U_{3} \xi_{0}^{2 \alpha-1} \frac{\cos (2 \alpha-1) \pi}{2}+\alpha U_{4} \xi_{0}^{\alpha-1} \frac{\cos (\alpha-1) \pi}{2}, \\
\mathscr{B}_{7}^{*}= & 5 \alpha \xi_{0}^{5 \alpha-1} \frac{\sin (5 \alpha-1) \pi}{2}+4 \alpha U_{1} \xi_{0}^{4 \alpha-1} \frac{\sin (4 \alpha-1) \pi}{2}+3 \alpha U_{2} \xi_{0}^{3 \alpha-1} \frac{\sin (3 \alpha-1) \pi}{2} \\
& +2 \alpha U_{3} \xi_{0}^{2 \alpha-1} \frac{\sin (2 \alpha-1) \pi}{2}+\alpha U_{4} \xi_{0}^{\alpha-1} \frac{\sin (\alpha-1) \pi}{2}, \\
\mathscr{A}_{8}^{*}= & 4 \alpha V_{1} \xi_{0}^{4 \alpha-1} \frac{\cos (4 \alpha-1) \pi}{2}+3 \alpha V_{2} \xi_{0}^{3 \alpha-1} \frac{\cos (3 \alpha-1) \pi}{2}+2 \alpha V_{3} \xi_{0}^{2 \alpha-1} \frac{\cos (2 \alpha-1) \pi}{2}+\alpha V_{4} \xi_{0}^{\alpha-1} \frac{\cos (\alpha-1) \pi}{2} \\
\mathscr{B}_{5}^{*}= & 4 \alpha V_{1} \xi_{0}^{4 \alpha-1} \frac{\sin (4 \alpha-1) \pi}{2}+3 \alpha V_{2} \xi_{0}^{3 \alpha-1} \frac{\sin (3 \alpha-1) \pi}{2}+2 \alpha V_{3} \xi_{0}^{2 \alpha-1} \frac{\sin (2 \alpha-1) \pi}{2}+\alpha V_{4} \xi_{0}^{\alpha-1} \frac{\sin (\alpha-1) \pi}{2}
\end{aligned}
$$


TABLE 1: $\xi_{0}$ values and $\tau_{1}^{*}$ values for different fractional-order $\alpha$.

\begin{tabular}{lcc}
\hline Fractional order $(\alpha)$ & Critical frequency $\left(\xi_{0}\right)$ & Bifurcation point $\left(\tau_{1}^{*}\right)$ \\
\hline 1 & 0.30125 & 1.2104 \\
0.9 & 0.17811 & 4.5874 \\
0.8 & 0.09392 & 11.8356 \\
0.7 & 0.04573 & 23.2562 \\
0.6 & 0.00152 & 744.420 \\
\hline
\end{tabular}

Case 4. $\tau_{1}=\tau_{2}=\tau_{3}=0$.

When $\tau_{1}=\tau_{2}=\tau_{3}=0$, the characteristic equation (18) becomes

$$
\omega^{5 \alpha}+Z_{1} \omega^{4 \alpha}+Z_{2} \omega^{3 \alpha}+Z_{3} \omega^{2 \alpha}+Z_{4} \omega^{\alpha}+Z_{5}=0
$$

Here,

$$
\begin{aligned}
& Z_{1}=2 a_{3}+a_{5}+2 a_{9}+a_{1}-a_{4}+a_{2}+a_{8} \\
& Z_{2}=a_{3}^{2}+4 a_{3} a_{9}+2 a_{3} a_{5}+2 a_{5} a_{9}+a_{9}^{2}+a_{1} a_{3}-2 a_{3} a_{4}+a_{1} a_{5}+2 a_{1} a_{9}-2 a_{4} a_{9}+a_{2} a_{3}+a_{2} a_{5}+2 a_{2} a_{9}+2 a_{3} a_{8} \\
& +a_{5} a_{8}+a_{8} a_{9}-a_{1} a_{4}+a_{2} a_{4}+a_{1} a_{4}-a_{2} a_{4}+a_{2} a_{8}-a_{4} a_{7}+a_{1} a_{8}-a_{4} a_{8} \\
& Z_{3}=2 a_{3} a_{9}^{2}+a_{5} a_{9}^{2}+2 a_{3}^{2} a_{9}+a_{3}^{2} a_{5}+4 a_{3} a_{5} a_{9}-a_{3}^{2} a_{4}+a_{1} a_{3} a_{5}+2 a_{1} a_{3} a_{9}-4 a_{3} a_{4} a_{9}+2 a_{1} a_{5} a_{9}+a_{1} a_{9}^{2}-a_{4} a_{9}^{2} \\
& +a_{2} a_{3} a_{5}+2 a_{2} a_{3} a_{9}+2 a_{2} a_{5} a_{9}+a_{2} a_{9}^{2}+a_{3}^{2} a_{8}+2 a_{3} a_{5} a_{8}+2 a_{3} a_{8} a_{9}+a_{5} a_{8} a_{9}-a_{1} a_{3} a_{4}-2 a_{1} a_{4} a_{9}+a_{2} a_{3} a_{4} \\
& +2 a_{2} a_{4} a_{9}+a_{1} a_{3} a_{4}-a_{2} a_{3} a_{4}+2 a_{1} a_{4} a_{9}-2 a_{2} a_{4} a_{9}+a_{2} a_{3} a_{8}+a_{2} a_{5} a_{8}+a_{2} a_{8} a_{9}-2 a_{3} a_{4} a_{7}+a_{1} a_{3} a_{8}-2 a_{3} a_{4} a_{8} \\
& +a_{1} a_{5} a_{8}-a_{4} a_{7} a_{9}+a_{1} a_{8} a_{9}-a_{4} a_{8} a_{9} \\
& Z_{4}=a_{3}^{2} a_{9}^{2}+2 a_{3} a_{5} a_{9}^{2}+2 a_{3}^{2} a_{5} a_{9}-2 a_{3}^{2} a_{4} a_{9}+2 a_{1} a_{3} a_{5} a_{9}+a_{1} a_{3} a_{9}^{2}-2 a_{3} a_{4} a_{9}^{2}+a_{1} a_{5} a_{9}^{2}+a_{2} a_{3} a_{9}^{2}+a_{2} a_{5} a_{9}^{2} \\
& +2 a_{2} a_{3} a_{5} a_{9}+a_{3}^{2} a_{5} a_{8}+a_{3}^{2} a_{8} a_{9}+2 a_{3} a_{5} a_{8} a_{9}-a_{1} a_{4} a_{9}^{2}-2 a_{1} a_{3} a_{4} a_{9}+2 a_{2} a_{3} a_{4} a_{9}+a_{1} a_{4} a_{9}^{2}-a_{2} a_{4} a_{9}^{2}+a_{2} a_{3} a_{5} a_{8} \\
& +a_{2} a_{3} a_{8} a_{9}+a_{2} a_{5} a_{8} a_{9}-a_{3}^{2} a_{4} a_{7}+a_{1} a_{3} a_{5} a_{8}-a_{3}^{2} a_{4} a_{8}-2 a_{3} a_{4} a_{7} a_{9}+a_{1} a_{3} a_{8} a_{9}-2 a_{3} a_{4} a_{8} a_{9}+a_{1} a_{5} a_{8} a_{9}-a_{1} a_{3} a_{4} a_{8} \\
& -a_{1} a_{4} a_{8} a_{9}+a_{2} a_{3} a_{4} a_{8}+a_{2} a_{4} a_{8} a_{9}+a_{1} a_{3} a_{4} a_{8}-a_{2} a_{3} a_{4} a_{8}+a_{1} a_{4} a_{8} a_{9}-a_{2} a_{4} a_{8} a_{9} \text {, } \\
& Z_{5}=a_{3}^{2} a_{5} a_{9}^{2}-a_{3}^{2} a_{4} a_{9}^{2}+a_{1} a_{3} a_{5} a_{9}^{2}+a_{2} a_{3} a_{5} a_{9}^{2}+a_{3}^{2} a_{5} a_{8} a_{9}-a_{1} a_{3} a_{4} a_{9}^{2}+a_{2} a_{3} a_{4} a_{9}^{2}-a_{2} a_{3} a_{4} a_{9}^{2}+a_{2} a_{3} a_{5} a_{8} a_{9}-a_{3}^{2} a_{4} a_{7} a_{9} \\
& +a_{1} a_{3} a_{5} a_{8} a_{9} \text {. }
\end{aligned}
$$

From the Routh-Hurwitz criteria, if we choose $Z_{i}>0, i=1,2,3,4,5, \quad Z_{1} Z_{2} Z_{3}>Z_{3}^{2}+Z_{1}^{2} Z_{4}$ and $\left(Z_{1} Z_{4}-\right.$ $\left.Z_{5}\right)\left(Z_{1} Z_{2} Z_{3}-Z_{3}^{2}-Z_{1}^{2} Z_{4}\right)>Z_{5}\left(Z_{1} Z_{2}-Z_{3}\right)^{2}+Z_{1} Z_{5}^{2}$, then the five eigenvalues of the characteristic equation (64) have negative real parts. Hence, the steady state fractional-order system (5) is asymptotically stable when $\tau_{1}=\tau_{2}=\tau_{3}=0$ (without time delays).

We arrive at the following theorem.

Theorem 1. If $\alpha \in(0,1]$ and an endemic equilibrium point $E^{*}$ exists for system (5), then the following results hold:

(i) When $\tau_{1}>0, \tau_{2}=0$, and $\tau_{3}=0$, the endemic steady state $E^{*}$ is asymptotically stable for $\tau_{1} \in\left[0, \tau_{1}^{*}\right)$ and the system undergoes a Hopf bifurcation at the origin at $\tau_{1}=\tau_{1}^{*}$ and the transversality condition holds, $\left.\operatorname{Re}\left(d s / d \tau_{1}\right)\right|_{\tau_{1}=\tau_{1}^{*}, \xi=\xi_{0}} \neq 0$

(ii) When $\tau_{1}=0, \tau_{2}>0$, and $\tau_{3}=0$, the endemic steady state $E^{*}$ is asymptotically stable for $\tau_{2} \in\left[0, \tau_{2}^{*}\right)$ and system undergoes a Hopf bifurcation at the origin when $\tau_{2}=\tau_{2}^{*}$ and the transversality condition holds, $\left.\operatorname{Re}\left(d s / d \tau_{2}\right)\right|_{\tau_{2}=\tau_{2}^{*}, \xi=\xi_{0}} \neq 0$

(iii) When $\tau_{1}=0, \tau_{2}=0$, and $\tau_{3}>0, E^{*}$ of is asymptotically stable for $\tau_{3} \in\left[0, \tau_{3}^{*}\right)$ and system (5) undergoes a Hopf bifurcation at the origin at $\tau_{3}=\tau_{3}^{*}$ and transversality condition holds, $\operatorname{Re}(d s)$ $\left.d \tau_{3}\right)\left.\right|_{\tau_{3}=\tau_{3}^{*}, \xi=\xi_{0}} \neq 0$

(iv) When $Z_{1} Z_{2} Z_{3}>Z_{3}^{2}+Z_{1}^{2} Z_{4}$ and $\left(Z_{1} Z_{4}-Z_{5}\right)$ $\left(Z_{1} Z_{2} Z_{3}-Z_{3}^{2}-Z_{1}^{2} Z_{4}\right)>Z_{5}\left(Z_{1} Z_{2}-Z_{3}\right)^{2}+Z_{1} Z_{5}^{2}$ holds, the endemic steady state $E^{*}$ is asymptotically stable for $\tau_{1}=\tau_{2}=\tau_{3}=0$

Remark 4. Theorem 1 reports the asymptotic stability of the endemic equilibrium point $E^{*}$. The analysis can be extended to investigate the stability of infection-free equilibrium points $E^{0}$ for the fractional-order model. 

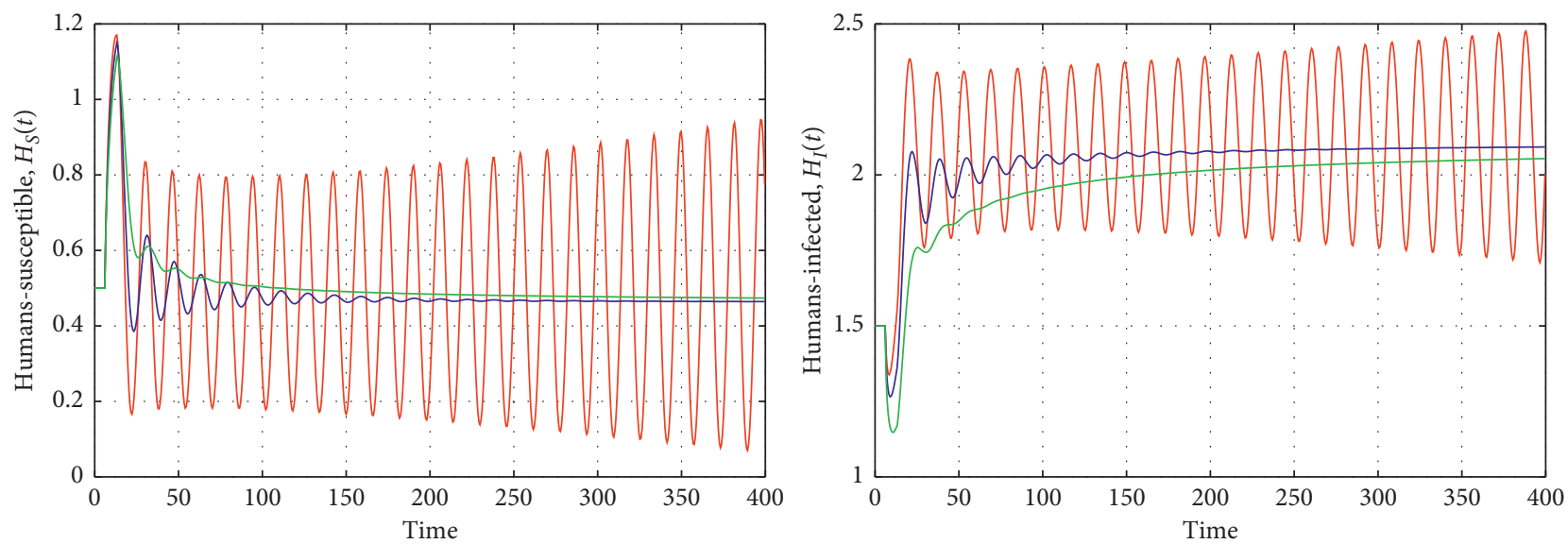

$$
\begin{array}{r}
\alpha=0.9 \\
-\alpha=0.8 \\
\alpha=0.7
\end{array}
$$$$
-\alpha=0.9
$$
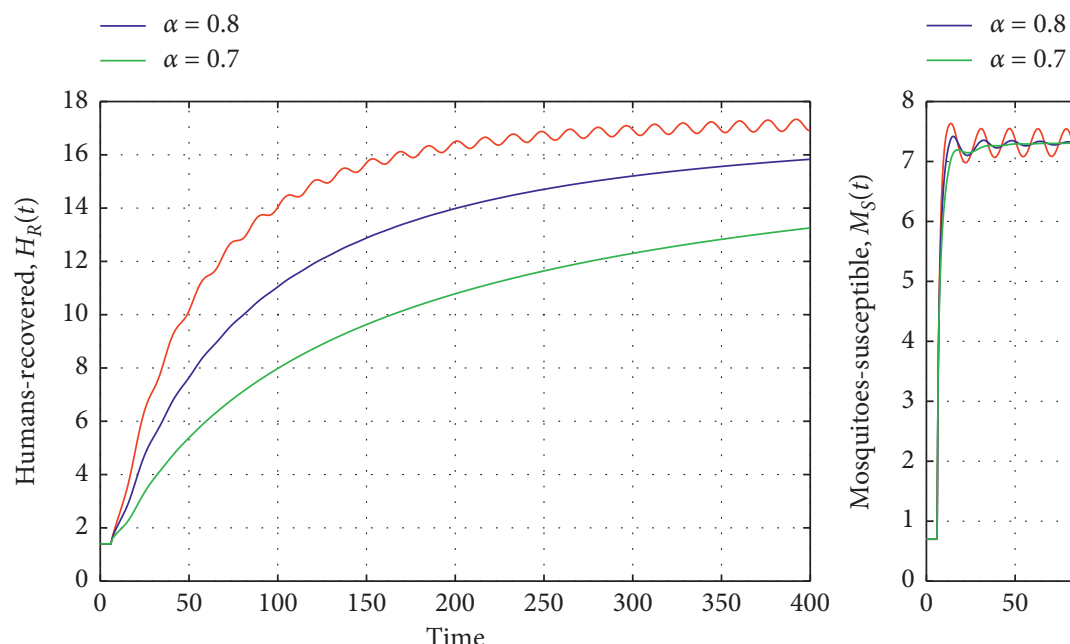

$$
-\alpha=0.7
$$
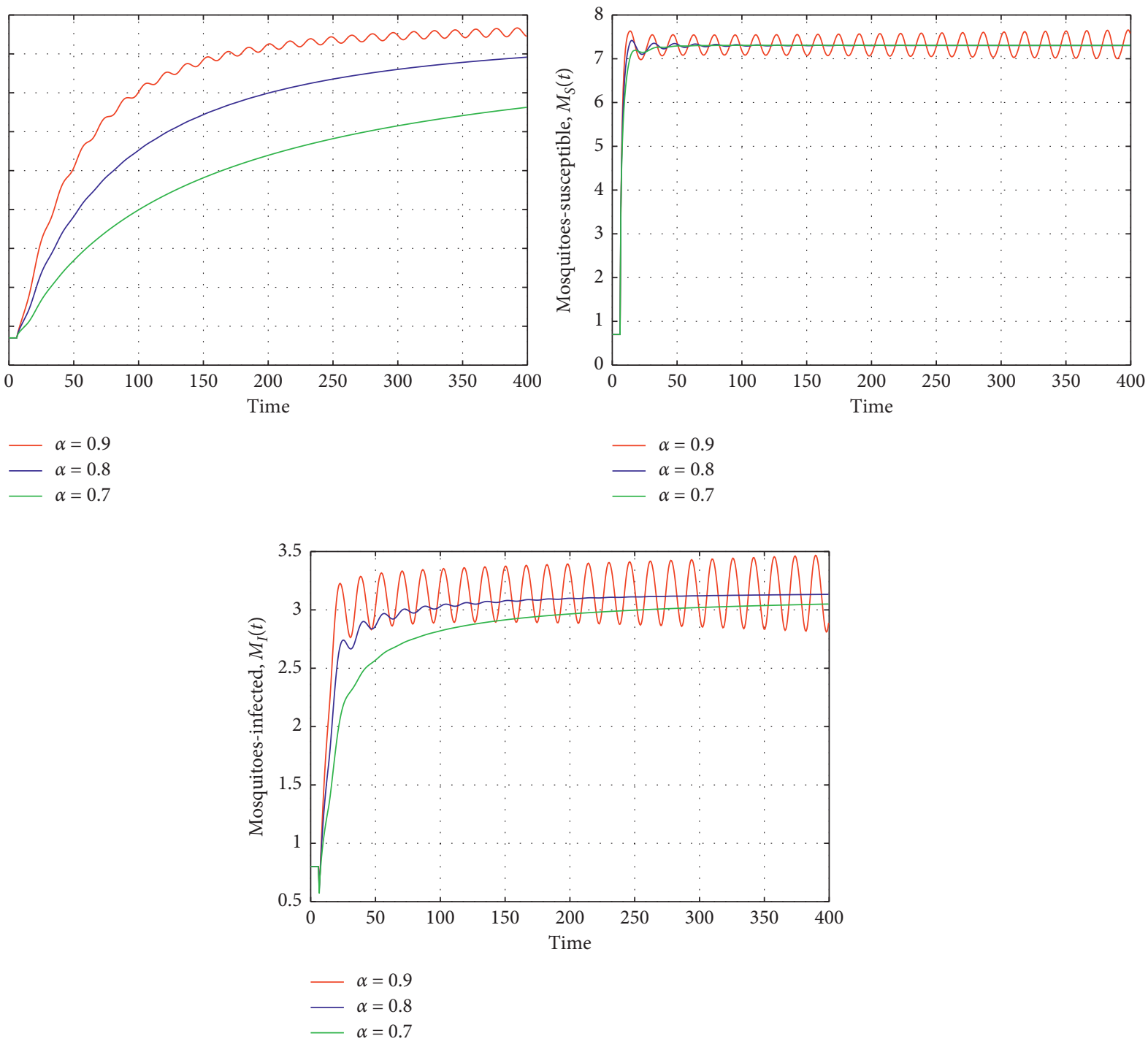

FIGURE 2: State trajectories for model (5) with $\alpha=0.9,0.8$, and 0.7 and $\tau_{1}=10, \tau_{2}=0.0$, and $\tau_{3}=0.0$. For $\alpha=0.9$ and $\tau_{1}=10>\tau_{1}^{*}$, the equilibrium point is unstable (red trajectory) for (5); however, for $\tau_{1}<\tau_{1}^{*}$ and $\alpha=0.8,0.7$, it is asymptotically stable (blue and green trajectories). 


\section{Numerical Simulations and Observations}

In this section, we provide some numerical simulations for system (5) to demonstrate the effectiveness of our main results. The simulations have been done by using stable implicit Euler approximation scheme, discussed in [44]. Of course, many other methods have been used for fractionalorder delay differential equations such as the AdamsBashforth-Moulton scheme [45]. The parameter values of system (5) are taken as follows:

$$
\begin{aligned}
\lambda_{h} & =0.5, \\
\lambda_{m} & =4.58, \\
\beta_{h} & =0.05, \\
\beta_{m} & =0.09, \\
\eta & =0.01, \\
\gamma & =0.2, \\
d_{h} & =0.714, \\
d_{m} & =0.437 .
\end{aligned}
$$

Case 1. $\tau_{1}>0, \tau_{2}=0$, and $\tau_{3}=0$. In this case, time-delay $\tau_{1}$ is chosen as the bifurcation parameter. We then discuss the dynamic effect of system (5) with the above parameter values. We calculate the critical frequency $\xi_{0}$ and bifurcation point $\tau_{1}^{*}$ of various fractional-order $\alpha$. Figure 2 shows the numerical simulations of model (5) when $\tau_{1}=10, \tau_{2}=0.0$, and $\tau_{3}=0.0$, with different fractional orders $\alpha=$ $0.9,0.8$, and 0.7 and estimated bifurcation point $\tau_{1}^{*}=4.587$, 11.835, and 23.256 (see Table 1 ). Here, $\tau_{1}=10 \notin\left[0, \tau_{1}^{*}\right)$ for the fractional-order $\alpha=0.9$ and whereas $\tau_{1}=10 \in\left[0, \tau_{1}^{*}\right)$ which satisfies the condition (i) in Theorem 1 . The equilibrium $E^{*}$ of the model (5) is asymptotically stable for $\alpha=0.8,0.7$. When $\tau_{1}=10 \notin\left[0, \tau_{1}^{*}\right)$, which does not satisfies the condition (i) of Theorem 1, the system undergoes a Hopf bifurcation for the functional-order $\alpha=0.9$.

Case 2. $\tau_{1}=0, \tau_{2}>0$, and $\tau_{3}=0$. We choose time-delay $\tau_{2}$ as a bifurcation parameter of system (5) with parameter values:

$$
\begin{aligned}
\lambda_{h} & =0.5, \\
\lambda_{m} & =4.58, \\
\beta_{h} & =0.05, \\
\beta_{m} & =0.08, \\
\eta & =0.05, \\
\gamma & =0.2, \\
d_{h} & =0.3, \\
d_{m} & =0.78 .
\end{aligned}
$$

We then calculate the critical frequency $\xi_{0}$ and bifurcation point $\tau_{2}^{*}$ of various fractional-order $\alpha$ (see Table 2). Figure 3 shows the dynamics of system (5) for $\tau_{1}=$ $0.0, \tau_{2}=14$, and $\tau_{3}=0.0$, with values of $\alpha=1,0.9$, and 0.8 .
TABLE 2: $\xi_{0}$ values and $\tau_{2}^{*}$ values for different fractional-order $\alpha$.

\begin{tabular}{lcc}
\hline Fractional order $(\alpha)$ & $\begin{array}{c}\text { Critical } \\
\text { frequency }\left(\xi_{0}\right)\end{array}$ & Bifurcation point $\left(\tau_{2}^{*}\right)$ \\
\hline 1 & 0.12290 & 11.1762 \\
0.9 & 0.09602 & 14.2931 \\
0.8 & 0.05174 & 35.797 \\
0.7 & 0.0363 & 47.963 \\
0.6 & 0.0158 & 131.001 \\
\hline
\end{tabular}

TABLE 3: $\xi_{0}$ values and $\tau_{3}^{*}$ values for different fractional-order $\alpha$.

\begin{tabular}{lcc}
\hline Fractional order $(\alpha)$ & $\begin{array}{c}\text { Critical } \\
\text { frequency }\left(\xi_{0}\right)\end{array}$ & Bifurcation point $\left(\tau_{3}^{*}\right)$ \\
\hline 1 & 0.3777 & 2.255 \\
0.9 & 0.2943 & 3.281 \\
0.8 & 0.2172 & 4.912 \\
0.7 & 0.01490 & 7.755 \\
0.6 & 0.0920 & 13.404 \\
\hline
\end{tabular}

The corresponding bifurcation point is $\tau_{2}^{*}=11.176$, 14.293, and 35.797. $\tau_{2}=14 \in\left[0, \tau_{2}^{*}\right)$ satisfies the condition (ii) of Theorem 1 . Therefore, the equilibrium $E^{*}$ of the model (5) is asymptotically stable for $\alpha=0.9$ and 0.8 , which is shown in Figure 3 . However, for $\tau_{2}=14 \notin\left[0, \tau_{2}^{*}\right)$, a Hopf bifurcation occurs for the functional-order $\alpha=1$.

Case 3. $\tau_{1}=0, \tau_{2}=0$, and $\tau_{3}>0$. We consider time-delay $\tau_{3}$ as a bifurcation parameter of system (5) with parameter values:

$$
\begin{aligned}
\lambda_{h} & =0.5, \\
\lambda_{m} & =10, \\
\beta_{h} & =0.05, \\
\beta_{m} & =0.4, \\
\eta & =0.05, \\
\gamma & =0.2, \\
d_{h} & =0.714, \\
d_{m} & =0.437 .
\end{aligned}
$$

We calculate the critical frequency $\xi_{0}$ and bifurcation point $\tau_{3}^{*}$ of various fractional-order $\alpha$. When $\tau_{1}=0.0$, $\tau_{2}=0.0$, and $\tau_{3}=8.0$, the dynamics of system (5) is shown in Figure 4 with different fractional-order $\alpha=1,0.9$, and 0.8 , its corresponding bifurcation points $\tau_{3}^{*}=2.255,3.281$, and 4.912 (see Table 1). Here, $\tau_{3}=8 \notin\left[0, \tau_{3}^{*}\right.$ ) and a Hopf bifurcation occurs for the factional-order $\alpha=1,0.9$, and 0.8 which not satisfies the condition (iii) in Theorem 1 . Therefore, the equilibrium point $E^{*}$ is of model (5) is unstable, which is shown in Figure 4.

Case 4. $\tau_{1}=0, \tau_{2}=0$, and $\tau_{3}=0$, without time delays. Assume the parameter values: 

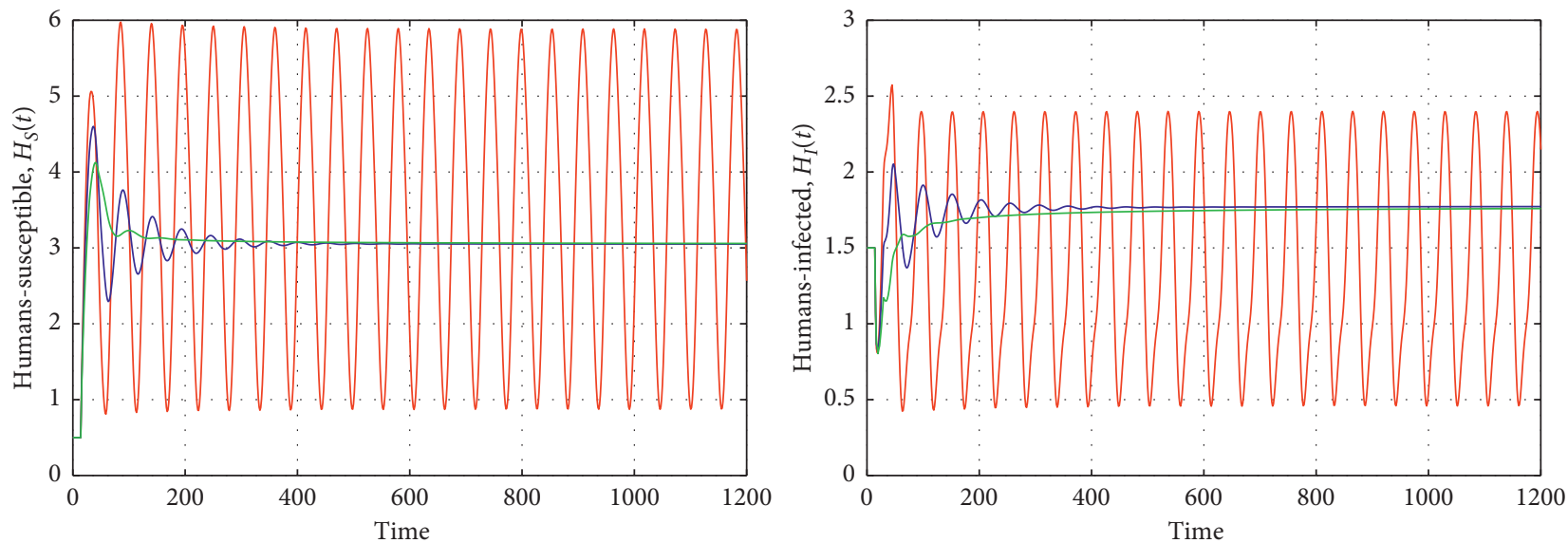

$-\alpha=1$

$-\alpha=1$

$-\alpha=0.9$

$-\alpha=0.9$

$-\alpha=0.8$

$-\alpha=0.8$
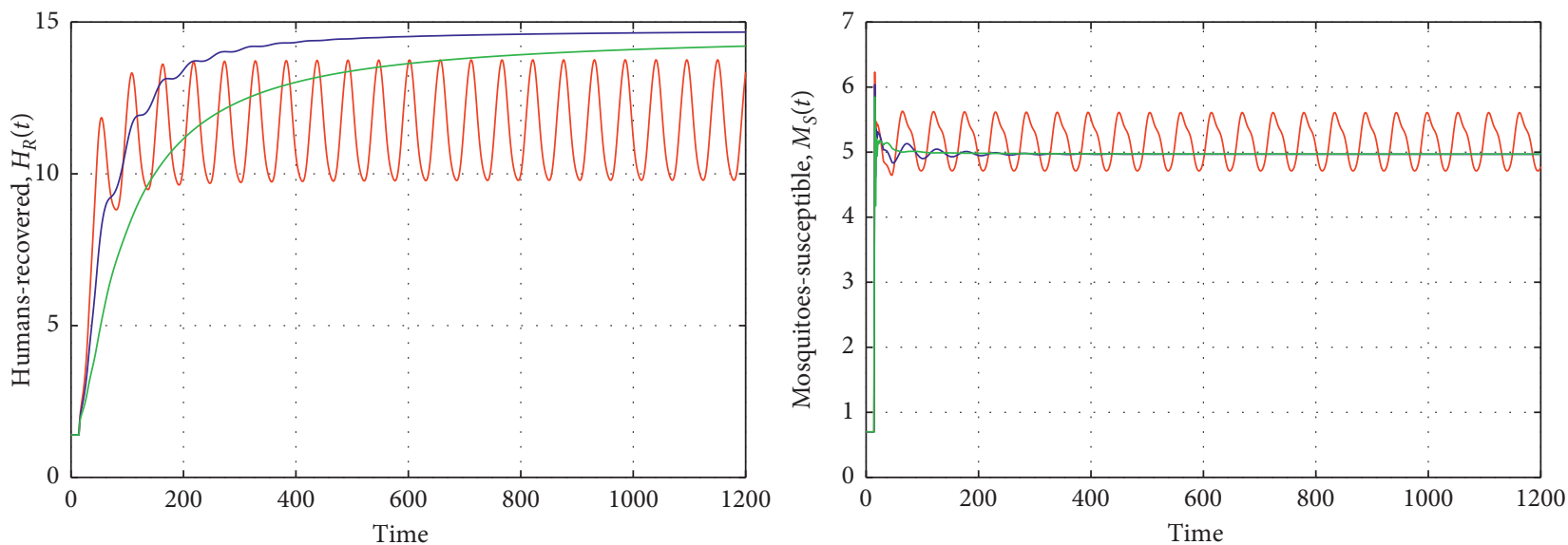

$-\alpha=1$

$\alpha=1$

$-\alpha=0.9$

$-\alpha=0.9$

$-\alpha=0.8$

$-\alpha=0.8$

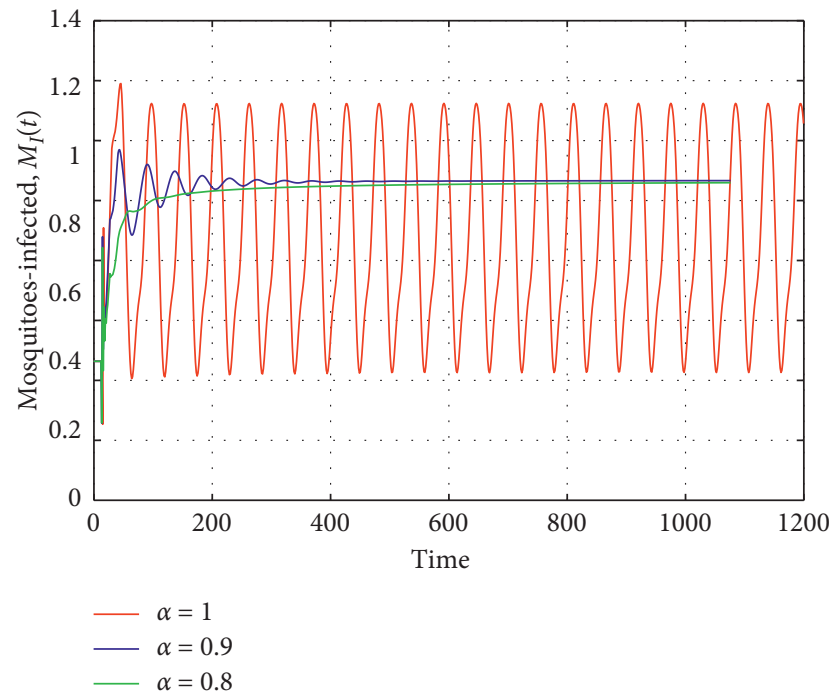

FIgURE 3: State trajectories for model (5) for $\alpha=1,0.9$, and 0.8 and $\tau_{1}=0.0, \tau_{2}=14$, and $\tau_{3}=0.0$, when $\alpha=1$ and $\tau_{2}=14>\tau_{2}^{*}$; the equilibrium point is unstable (red trajectory) for (5). However, for $\tau_{2}<\tau_{2}^{*}$ with $\alpha=0.9$ and 0.8 , it is asymptotically stable (blue and green trajectories). 

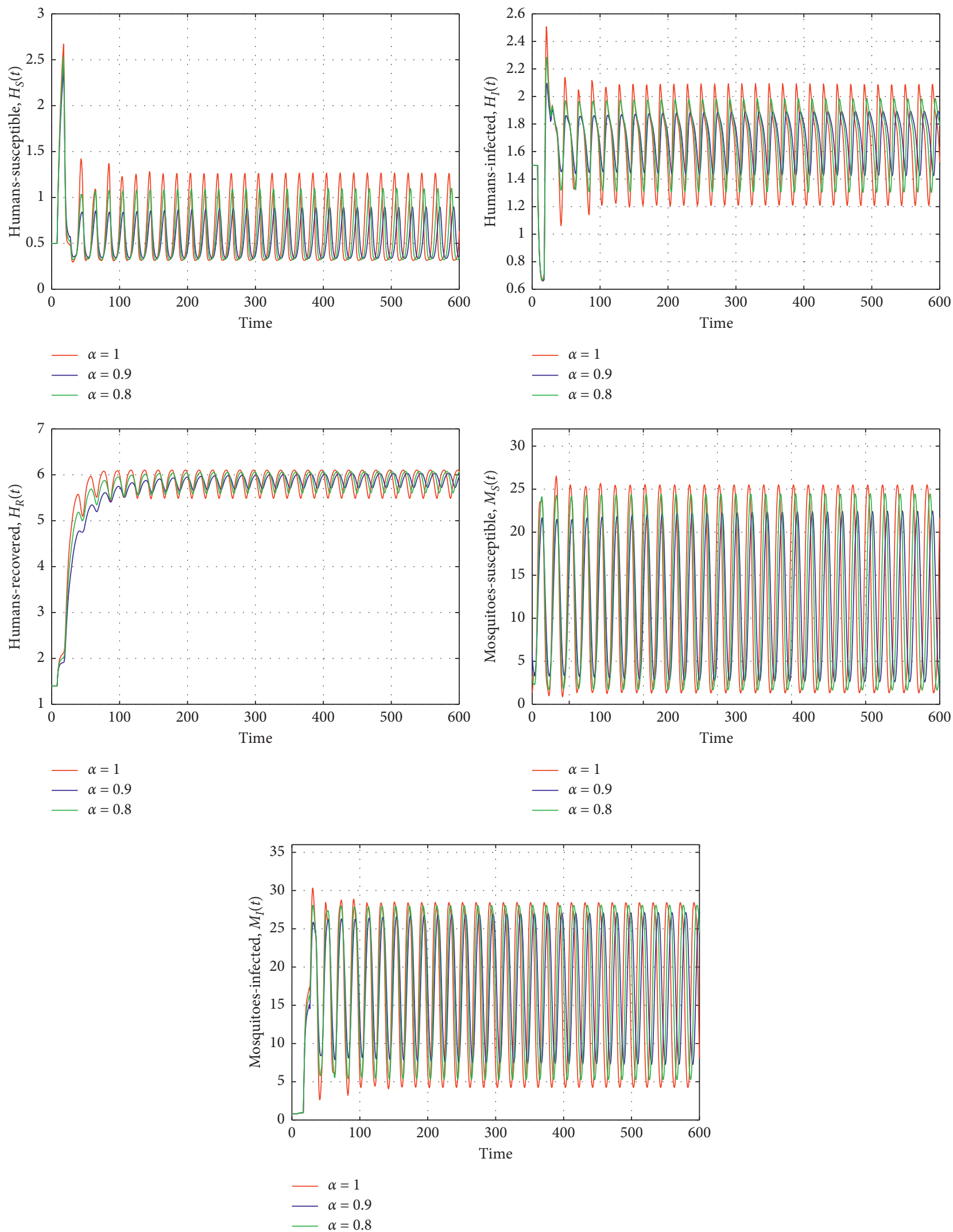

FIgURE 4: State trajectories for the model (5) for various values of $\alpha=1,0.9$, and 0.8 and $\tau_{1}=0.0, \tau_{2}=0.0$, and $\tau_{3}=8$. The equilibrium point $E^{*}$ is unstable when $\tau_{3} \notin\left[0, \tau_{3}^{*}\right)$. 

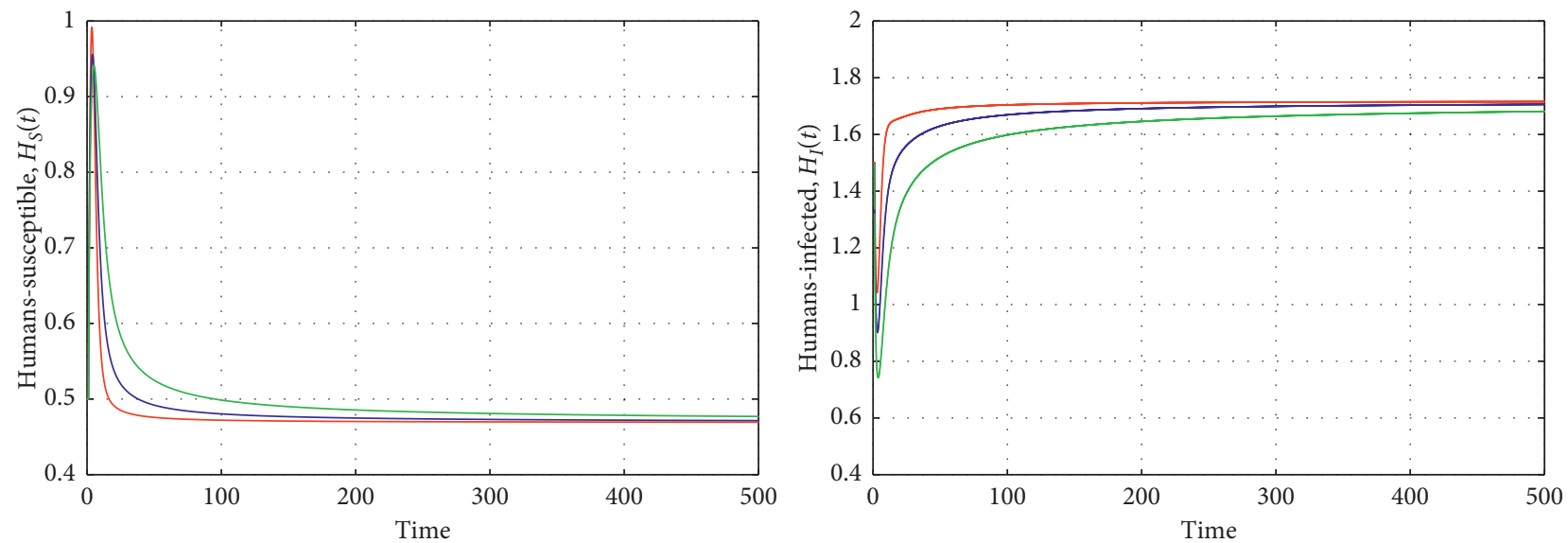

$\begin{aligned} \alpha & =0.9 \\ \alpha & =0.8\end{aligned}$

$-\alpha=0.7$

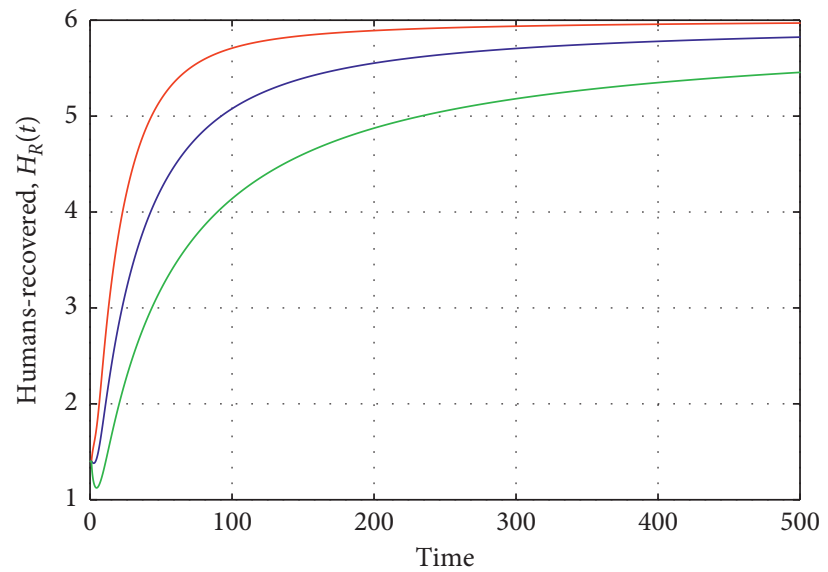

$$
\begin{array}{r}
\alpha=0.9 \\
-\alpha=0.8 \\
\alpha=0.7
\end{array}
$$

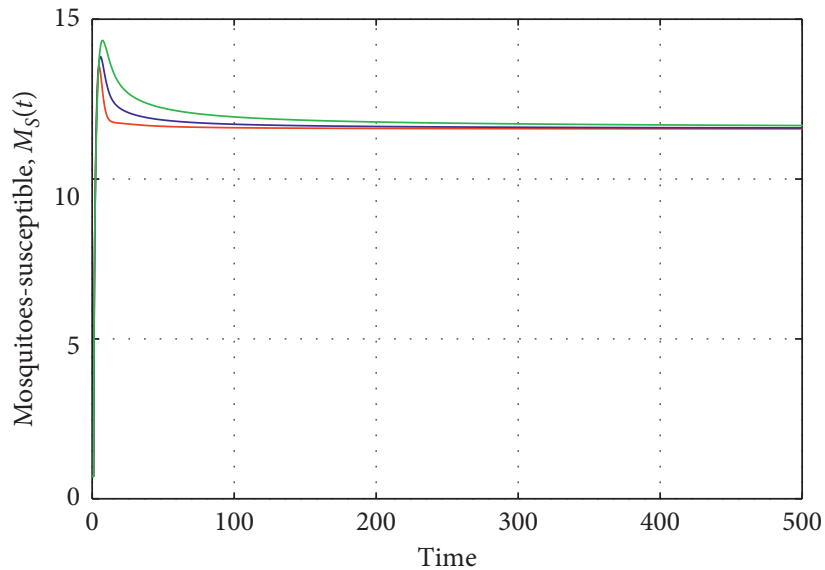

$$
\begin{aligned}
\alpha & =0.9 \\
\alpha & =0.8 \\
\alpha & =0.7
\end{aligned}
$$$$
-\alpha=0.9
$$

$-\alpha=0.8$

$-\alpha=0.7$

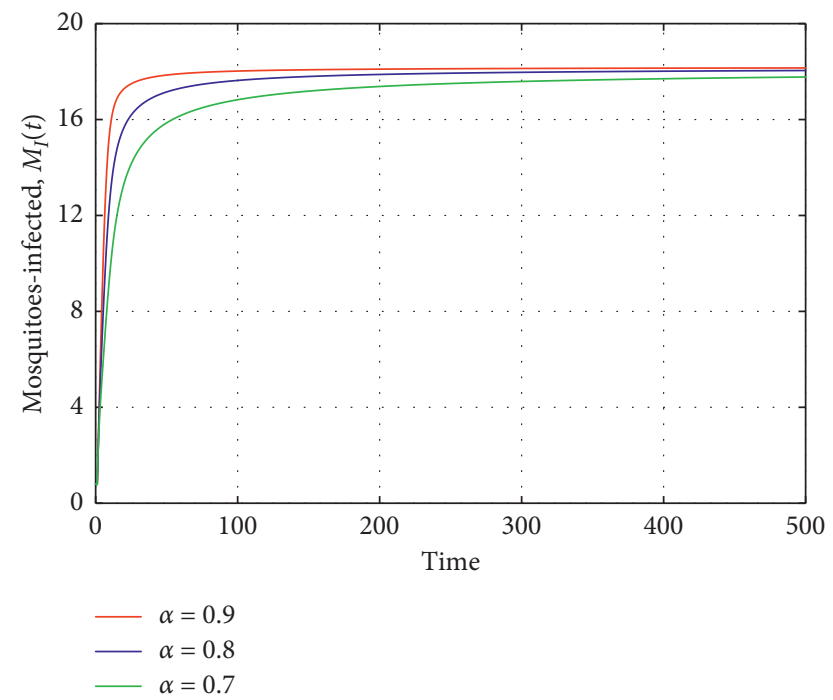

Figure 5: State trajectories of model (5) for various values of $\alpha=0.9,0.8$, and 0.7 , when $\tau_{1}=\tau_{2}=\tau_{3}=0$. The steady state of the system is asymptotically stable. 


$$
\begin{aligned}
\lambda_{h} & =0.5, \\
\lambda_{m} & =10, \\
\beta_{h} & =0.05, \\
\beta_{m} & =0.4, \\
\eta & =0.05, \\
\gamma & =0.2, \\
d_{h} & =0.714, \\
d_{m} & =0.437 .
\end{aligned}
$$

Hence, system (5) is asymptotically stable, which is shown in Figure 5.

\section{Conclusion}

Fractional derivatives have the unique property of capturing the history of the variable; that is, they have short and long memory. This cannot be easily done by means of the integerorder derivatives. In this paper, we proposed a fractionalorder model for Zika virus infection with multiple time delays $\tau_{1}, \tau_{2}$, and $\tau_{3}$. We studied the asymptotic stability and Hopf bifurcation properties for the model. Time delays and fractional order play a vital role in the stability and complexity of the model. By evaluating the characteristics, some sufficient conditions have derived to ensure the asymptotic stability in terms of the fractional order and time delays. Moreover, we estimated the thresholds bifurcation parameters: $\tau_{1}^{*}, \tau_{2}^{*}$, and $\tau_{3}^{*}$. The transversality conditions have been obtained to confirm the existence of Hopf bifurcations for different values at the threshold parameters and particular values of fractional orders. Our findings illustrate that using the time delays as bifurcation points, one can conclude that when time delay increases, the equilibrium loses its stability and Hopf bifurcation occurs. These models can be used to understand key aspects of the viral life cycle and to predict antiviral efficacy. Finally, numerical simulations show that a combination of fractional order and time delays in the model effectively enriches the dynamics and strengthens the stability condition of the model.

Including control variables in the model is desirable to determine the best strategy of treatment and control and eliminate the infection, which will be considered in future work.

\section{Appendix}

The coefficients of equation (18) are as follows:

$$
\begin{aligned}
& \mathbb{P}_{1}(s)=s^{5 \alpha}+s^{4 \alpha}\left(a_{3}+2 a_{9}\right)+s^{3 \alpha}\left(a_{3}^{2}+2 a_{3} a_{5}+2 a_{5} a_{9}+a_{9}^{2}\right)+s^{2 \alpha}\left(a_{3}^{2} a_{5}+4 a_{3} a_{5} a_{9}+2 a_{3} a_{9}^{2}+a_{5} a_{9}^{2}\right) \\
& +s^{\alpha}\left(2 a_{3}^{2} a_{5} a_{9}+2 a_{3} a_{5} a_{9}^{2}\right)+a_{3}^{2} a_{9}^{2} \text {, } \\
& \mathbb{P}_{2}(s)=s^{4 \alpha} a_{1}+s^{3 \alpha}\left(a_{1} a_{3}+a_{1} a_{5}+2 a_{1} a_{9}\right)+s^{2 \alpha}\left(a_{1} a_{3} a_{5}+2 a_{1} a_{3} a_{9}+2 a_{1} a_{5} a_{9}+a_{1} a_{9}^{2}\right)+s^{\alpha}\left(2 a_{1} a_{3} a_{5} a_{9}+a_{1} a_{3} a_{9}^{2}+a_{1} a_{5} a_{9}^{2}\right) \\
& +a_{1} a_{3} a_{5} a_{9}^{2} \\
& \mathbb{P}_{3}(s)=s^{4 \alpha}\left(a_{2}-a_{4}\right)+s^{3 \alpha}\left(a_{2} a_{3}-2 a_{3} a_{4}+a_{2} a_{5}+2 a_{2} a_{9}-2 a_{4} a_{9}\right)+s^{2 \alpha}\left(-a_{3}^{2} a_{4}+a_{2} a_{3} a_{5}+2 a_{2} a_{3} a_{9}-4 a_{3} a_{4} a_{9}+a_{2} a_{5} a_{9}\right. \\
& \left.+a_{2} a_{9}^{2}-a_{4} a_{9}^{2}\right)+s^{\alpha}\left(-2 a_{3}^{2} a_{4} a_{9}+2 a_{2} a_{3} a_{5} a_{9}+a_{2} a_{3} a_{9}^{2}-2 a_{3} a_{4} a_{9}^{2}+a_{2} a_{5} a_{9}^{2}\right)+a_{2} a_{3} a_{5} a_{9}^{2}-a_{3}^{2} a_{4} a_{9}^{2}, \\
& \mathbb{P}_{4}(s)=s^{4 \alpha} a_{8}+s^{3 \alpha}\left(2 a_{3} a_{8}+a_{5} a_{8}+a_{8} a_{9}\right)+s^{2 \alpha}\left(a_{3}^{2} a_{8}+2 a_{3} a_{5} a_{8}+2 a_{3} a_{8} a_{9}+a_{5} a_{8} a_{9}\right)+s^{\alpha}\left(a_{3}^{2} a_{5} a_{8}+a_{3}^{2} a_{8} a_{9}+a_{3} a_{5} a_{8} a_{9}\right) \\
& +a_{3}^{2} a_{5} a_{8} a_{9} \\
& \mathbb{P}_{5}(s)=s^{3 \alpha}\left(a_{1} a_{4}\right)+s^{2 \alpha}\left(a_{1} a_{3} a_{4}-2 a_{1} a_{4} a_{9}-a_{1} a_{3} a_{5} a_{9}\right)+s^{\alpha}\left(a_{1} a_{3} a_{4} a_{7}+a_{1} a_{4} a_{9}^{2}\right), \\
& \mathbb{P}_{6}(s)=s^{3 \alpha}\left(a_{2} a_{4}\right)+s^{2 \alpha}\left(a_{2} a_{3} a_{4}+2 a_{2} a_{4} a_{9}\right)+s^{\alpha}\left(a_{2} a_{4} a_{9}^{2}+a_{2} a_{3} a_{4} a_{9}\right)+a_{2} a_{3} a_{4} a_{9}^{2} \text {, } \\
& \mathbb{P}_{7}(s)=s^{4 \alpha}\left(-a_{4}\right)+s^{3 \alpha}\left(-a_{2} a_{4}+a_{1} a_{8}-a_{4} a_{7}\right)+s^{2 \alpha}\left(a_{2} a_{3} a_{5}+2 a_{1} a_{5} a_{9}+a_{4} a_{9}^{2}\right)+s^{\alpha}\left(a_{2} a_{9}^{2}+a_{1} a_{4} a_{9}^{2}\right)+a_{1} a_{3} a_{4} a_{9}^{2}-a_{2} a_{3} a_{4} a_{9}^{2}, \\
& \mathbb{P}_{8}(s)=s^{3 \alpha}\left(-a_{4} a_{7}+a_{2} a_{8}-a_{4} a_{8}\right)+s^{2 \alpha}\left(-2 a_{3} a_{4} a_{7}+a_{2} a_{3} a_{8}+a_{3} a_{4} a_{8}+a_{2} a_{5} a_{8}-a_{4} a_{7} a_{9}+a_{2} a_{8} a_{9}+a_{4} a_{8} a_{9}\right) \\
& +s^{\alpha}\left(-a_{3}^{2} a_{4} a_{7}-a_{3}^{2} a_{4} a_{8}-2 a_{3} a_{4} a_{7} a_{9}-+a_{2} a_{3} a_{8} a_{9}-2 a_{3} a_{4} a_{8} a_{9}+a_{2} a_{5} a_{8} a_{9}\right)-a_{3}^{2} a_{4} a_{7} a_{9}+a_{2} a_{3} a_{5} a_{8} a_{9}, \\
& \mathbb{P}_{9}(s)=s^{3 \alpha} a_{1} a_{8}+s^{2 \alpha}\left(a_{1} a_{4} a_{7}+a_{1} a_{3} a_{8}+a_{1} a_{5} a_{8}+a_{1} a_{8} a_{9}\right)+s^{\alpha}\left(a_{1} a_{3} a_{5} a_{8}+a_{1} a_{3} a_{8} a_{9}+a_{1} a_{5} a_{8} a_{9}\right)+a_{1} a_{3} a_{5} a_{7} a_{9} \text {, } \\
& \mathbb{P}_{10}(s)=s^{\alpha}\left(a_{1} a_{3} a_{4} a_{7}+a_{1} a_{4} a_{7} a_{9}\right)+a_{1} a_{3} a_{4} a_{7} a_{9} \\
& \mathbb{P}_{11}(s)=s^{\alpha}\left(-a_{2} a_{4} a_{7}-a_{2} a_{3} a_{4} a_{7}-a_{2} a_{4} a_{7} a_{9}\right)-a_{2} a_{3} a_{4} a_{7} a_{9} \\
& \mathbb{P}_{12}(s)=s^{2 \alpha}\left(-a_{1} a_{4} a_{7}+a_{2} a_{4} a_{7}\right)+s^{\alpha}\left(-a_{1} a_{3} a_{4} a_{7}+a_{2} a_{3} a_{4} a_{7}-a_{1} a_{4} a_{7} a_{9}+a_{2} a_{4} a_{7} a_{9}\right)-a_{1} a_{3} a_{7} a_{9}+a_{2} a_{3} a_{4} a_{8} a_{9} \text {. }
\end{aligned}
$$




\section{Data Availability}

Data sharing is not applicable to this article as no new data were created or analyzed in this study.

\section{Conflicts of Interest}

The authors declare that they have no conflicts of interest.

\section{Acknowledgments}

This manuscript was funded from the project no. $31 \mathrm{~S} 265$ (UAE University) and DST-SERB Project \# SB/FTP/MS045/2013 (Bharathiar University).

\section{References}

[1] G. Calvet, R. S. Aguiar, A. S. O. Melo et al., "Detection and sequencing of zika virus from amniotic fluid of fetuses with microcephaly in Brazil: a case study," The Lancet Infectious Diseases, vol. 16, no. 6, pp. 653-660, 2016.

[2] F. Krauer, M. Riesen, L. Reveiz et al., "Zika virus infection as a cause of congenital brain abnormalities and Guillain-Barré syndrome: systematic review," PLoS Medicine, vol. 14, no. 1, Article ID e1002203, 2017.

[3] E. Bonyah and K. O. Okosun, "Mathematical modeling of zika virus," Asian Pacific Journal of Tropical Disease, vol. 6, no. 9, pp. 673-679, 2016.

[4] D. Gao, Y. Lou, D. He et al., "Prevention and control of zika as a mosquito-borne and sexually transmitted disease: a mathematical modeling analysis," Scientific Reports, vol. 6, no. 1, Article ID 28070, 2016.

[5] F. A. Rihan, S. Lakshmanan, and H. Maurer, "Optimal control of tumour-immune model with time-delay and immunochemotherapy," Applied Mathematics and Computation, vol. 353, no. 7, pp. 147-165, 2019.

[6] N. MacDonald, Biological Delay Systems, Cambridge University Press, Cambridge, UK, 1989.

[7] N. Burić and D. Todorović, "Dynamics of delay-differential equations modelling immunology of tumor growth," Chaos, Solitons \& Fractals, vol. 13, no. 4, pp. 645-655, 2002.

[8] S. Gao, L. Chen, and Z. Teng, "Pulse vaccination of an SEIR epidemic model with time delay," Nonlinear Analysis: Real World Applications, vol. 9, no. 2, pp. 599-607, 2008.

[9] M. Y. Li and H. Shu, "Global dynamics of an in-host viral model with intracellular delay," Bulletin of Mathematical Biology, vol. 72, no. 6, pp. 1492-1505, 2010.

[10] F. A. Rihan and D. H. Abdel Rahman, "Delay differential model for tumour-immune dynamics with HIV infection of $\mathrm{CD}^{+}$T-cells," International Journal of Computer Mathematics, vol. 90, no. 3, pp. 594-614, 2013.

[11] K. Wang, W. Wang, H. Pang, and X. Liu, "Complex dynamic behavior in a viral model with delayed immune response," Physica D: Nonlinear Phenomena, vol. 226, no. 2, pp. 197-208, 2007.

[12] H.-M. Wei, X.-Z. Li, and M. Martcheva, "An epidemic model of a vector-borne disease with direct transmission and time delay," Journal of Mathematical Analysis and Applications, vol. 342, no. 2, pp. 895-908, 2008.

[13] W. Sakdanupaph, E. J. Moore, G. Maroulis, and T. E. Simos, "A delay differential equation model for dengue fever transmission in selected countries of south-east asia," AIP Conference Proceedings, vol. 1148, pp. 816-819, 2009.
[14] C. Chiyaka, J. M. Tchuenche, W. Garira, and S. Dube, "A mathematical analysis of the effects of control strategies on the transmission dynamics of malaria," Applied Mathematics and Computation, vol. 195, no. 2, pp. 641-662, 2008.

[15] W.-C. Chen, "Nonlinear dynamics and chaos in a fractionalorder financial system," Chaos, Solitons \& Fractals, vol. 36, no. 5, pp. 1305-1314, 2008.

[16] K. S. Cole, "Electric conductance of biological systems," Cold Spring Harbor Symposia on Quantitative Biology, vol. 1, pp. 107-116, 1933.

[17] R. Hilfer, Applications of Fractional Calculus in Physics, World Scientific, Singapore, 2000.

[18] H. Sheng, Y. Chen, and T. Qiu, Fractional Processes and Fractional-Order Signal Processing: Techniques and Applications, Springer, New York, NY, USA, 2012.

[19] C. A. Pinto and A. M. Carvalho, "Fractional dynamics of an infection model with time-varying drug exposure," Journal of Computational and Nonlinear Dynamics, vol. 13, no. 9, p. 16, 2018.

[20] G. Zaslavsky, "Chaos, fractional kinetics, and anomalous transport," Physics Reports, vol. 371, no. 6, pp. 461-580, 2002.

[21] F. A. Rihan, M. Sheek-Hussein, A. Tridane, and R. Yafia, "Dynamics of hepatitis C virus infection: mathematical modeling and parameter estimation," Mathematical Modelling of Natural Phenomena, vol. 12, no. 5, pp. 33-47, 2017.

[22] E. Ahmed, A. Hashish, and F. A. Rihan, "On fractional order cancer model," Journal of Fractional Calculus and Applied Analysis, vol. 3, no. 2, pp. 1-6, 2012.

[23] C. L. Althaus, V. V. Ganusov, and R. J. De Boer, "Dynamics of $\mathrm{CD}^{+} \mathrm{T}$ cell responses during acute and chronic lymphocytic choriomeningitis virus infection," The Journal of Immunology, vol. 179, no. 5, pp. 2944-2951, 2007.

[24] F. A. Rihan, D. Baleanu, S. Lakshmanan, and R. Rakkiyappan, "On fractional SIRC model with salmonella bacterial infection," Abstract and Applied Analysis, vol. 2014, Article ID 136263, 9 pages, 2014.

[25] L. Zhang, G. Huang, A. Liu, and R. Fan, "Stability analysis for a fractional HIV infection model with nonlinear incidence," Discrete Dynamics in Nature and Society, vol. 2015, Article ID 563127, 11 pages, 2015.

[26] V. P. Latha, F. A. Rihan, R. Rakkiyappan, and G. Velmurugan, "A fractional-order model for ebola virus infection with delayed immune response on heterogeneous complex networks," Journal of Computational and Applied Mathematics, vol. 339, pp. 134-146, 2018.

[27] V. P. Latha, F. A. Rihan, R. Rakkiyappan, and G. Velmurugan, "A fractional-order delay differential model for ebola infection and $\mathrm{CD}^{+} \mathrm{T}$-cells response: stability analysis and hopf bifurcation," International Journal of Biomathematics, vol. 10, no. 8, Article ID 1750111, 2017.

[28] A. J. Kucharski, S. Funk, R. M. Eggo et al., "Transmission dynamics of zika virus in island populations: a modelling analysis of the 2013-14 French polynesia outbreak," PLoS Neglected Tropical Diseases, vol. 10, no. 5, Article ID e0004726, 2016.

[29] V. M. Moreno, B. Espinoza, D. Bichara, S. A. Holechek, and C. Castillo-Chavez, "Role of short-term dispersal on the dynamics of zika virus in an extreme idealized environment," Infectious Disease Modelling, vol. 2, no. 1, pp. 21-34, 2017.

[30] S. Usman, I. I. Adamu, and U. Dahiru, "Stability analysis of a mathematical model for the transmission dynamics of zika virus infection," Journal of the Nigerian Association of Mathematical Physics, vol. 40, 2017. 
[31] F. Brauer, C. Castillo-Chavez, and Z. Feng, "Dengue fever and the zika virus," in Texts in Applied Mathematics, pp. 409-425, Springer, Berlin, Germany, 2019.

[32] X. Wang, Z. Wang, and H. Shen, "Dynamical analysis of a discrete-time sis epidemic model on complex networks," Applied Mathematics Letters, vol. 94, pp. 292-299, 2019.

[33] Z. Wang, Y. Xie, J. Lu, and Y. Li, "Stability and bifurcation of a delayed generalized fractional-order prey-predator model with interspecific competition," Applied Mathematics and Computation, vol. 347, pp. 360-369, 2019.

[34] X. Wang, Z. Wang, X. Huang, and Y. Li, "Dynamic analysis of a delayed fractional-order sir model with saturated incidence and treatment functions," International Journal of Bifurcation and Chaos, vol. 28, no. 14, Article ID 1850180, 2018.

[35] X. Wang, Z. Wang, and J. Xia, "Stability and bifurcation control of a delayed fractional-order eco-epidemiological model with incommensurate orders," Journal of the Franklin Institute, vol. 356, no. 15, pp. 8278-8295, 2019.

[36] F. A. Rihan, S. Lakshmanan, A. H. Hashish, R. Rakkiyappan, and E. Ahmed, "Fractional-order delayed predator-prey systems with Holling type-II functional response," Nonlinear Dynamics, vol. 80, no. 1-2, pp. 777-789, 2015.

[37] A. A. Tateishi, H. V. Ribeiro, and E. K. Lenzi, "The role of fractional time-derivative operators on anomalous diffusion," Frontiers in Physics, vol. 5, no. 1-9, 2017.

[38] A. Atangana, "Blind in a commutative world: simple illustrations with functions and chaotic attractors," Chaos, Solitons \& Fractals, vol. 114, pp. 347-363, 2018.

[39] A. Atangana, "Non validity of index law in fractional calculus: a fractional differential operator with Markovian and nonMarkovian properties," Physica A: Statistical Mechanics and Its Applications, vol. 505, pp. 688-706, 2018.

[40] A. Atangana and J. F. Gómez-Aguilar, "Decolonisation of fractional calculus rules: breaking commutativity and associativity to capture more natural phenomena," European Physical Journal Plus, vol. 133, no. 4, p. 166, 2018.

[41] I. Podlubny, Fractional Differential Equations, Academic Press, Cambridge, MA, USA, 1999.

[42] R. M. Anderson and R. M. May, Infectious Diseases of Humans: Dynamics and Control, Oxford University Press, Oxford, UK, 1992.

[43] E. J. Muth, Transform Methods: with Applications to Engineering and Operations Research, Prentice-Hall, Upper Saddle River, NJ, USA, 1977.

[44] F. A. Rihan, "Numerical modeling of fractional-order biological systems," Abstract and Applied Analysis, vol. 2013, Article ID 816803, 11 pages, 2013.

[45] B. P. Moghaddam, S. Yaghoobi, and J. A. Tenreiro Machado, "An extended predictor-corrector algorithm for variable-order fractional delay differential equations," Journal of Computational and Nonlinear Dynamics, vol. 11, no. 6, Article ID 061001, 7 pages, 2016. 


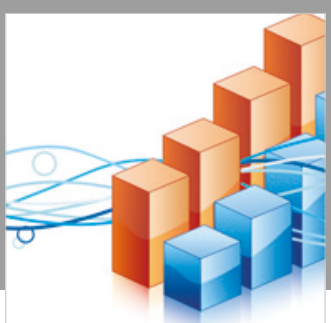

Advances in

Operations Research

\section{-n-m}
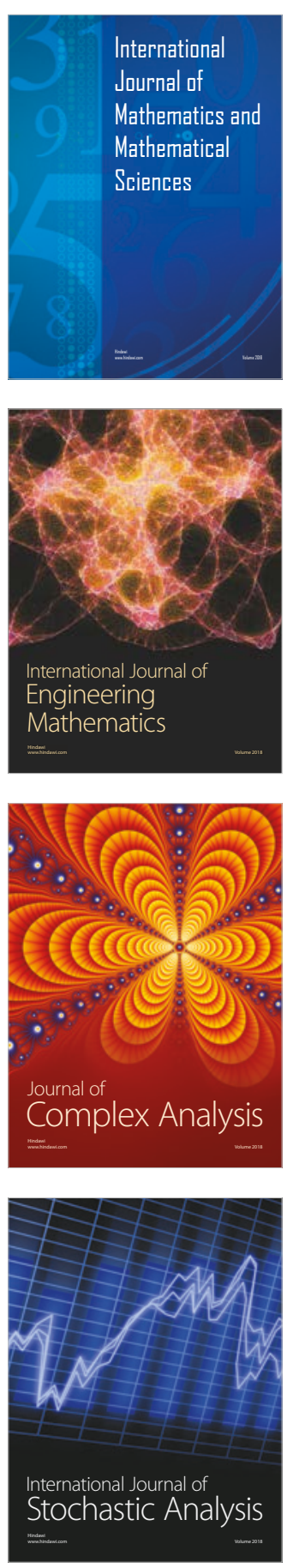
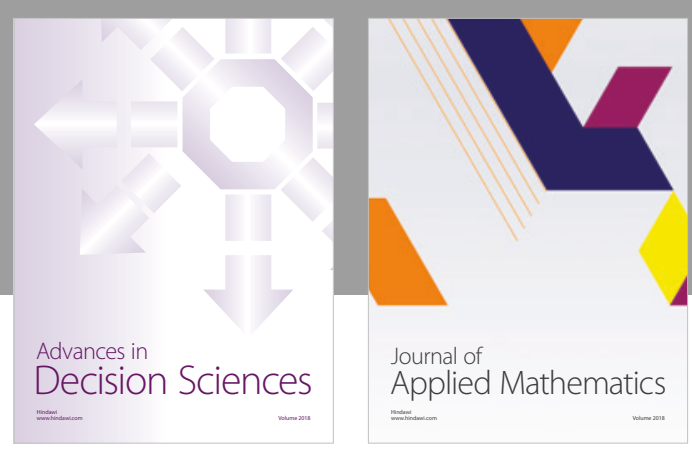

Journal of

Applied Mathematics
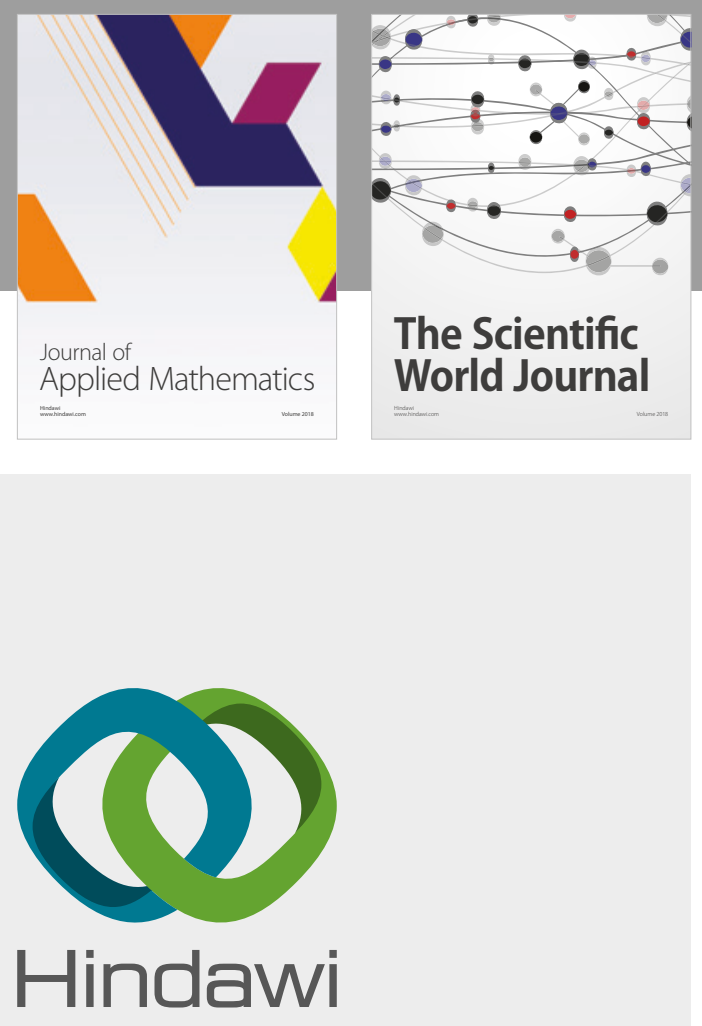

Submit your manuscripts at

www.hindawi.com

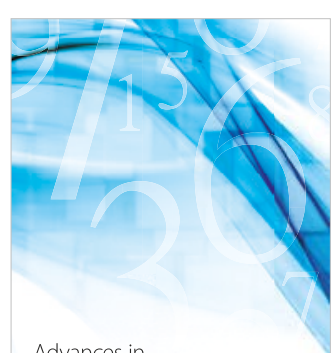

Advances in
Numerical Analysis
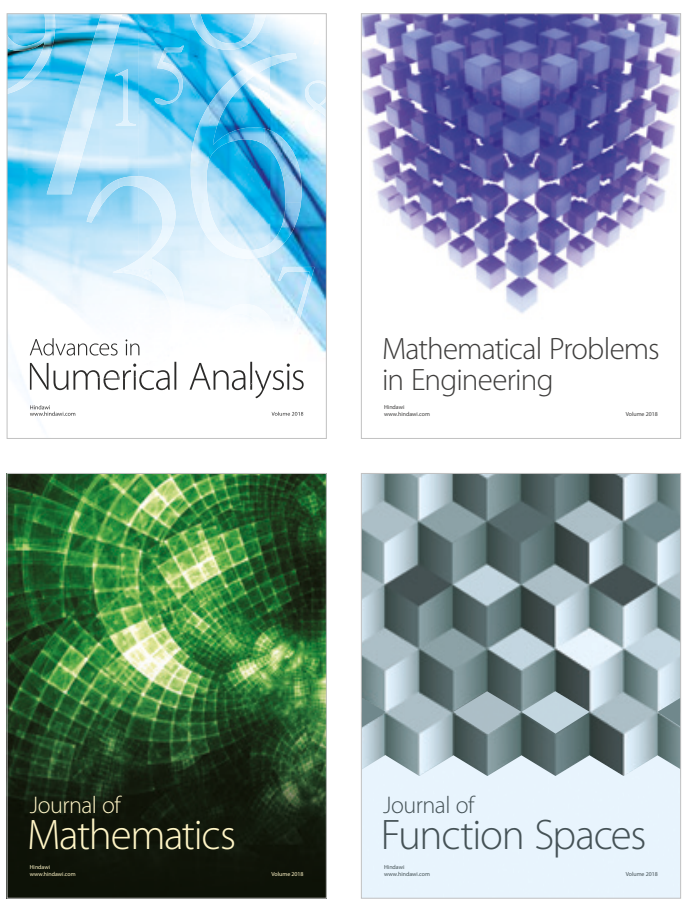

Mathematical Problems in Engineering

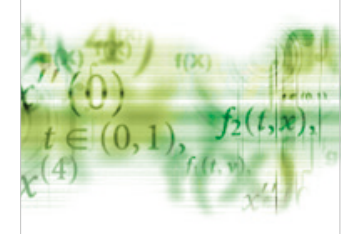

International Journal of

Differential Equations

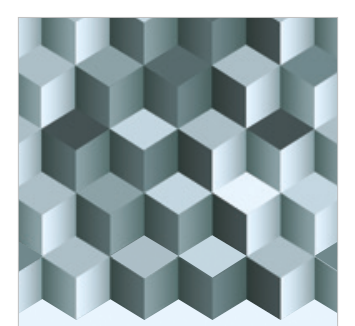

Journal of

Function Spaces
The Scientific

World Journal

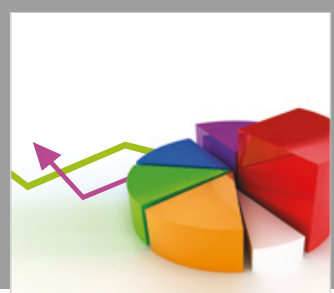

Journal of

Probability and Statistics
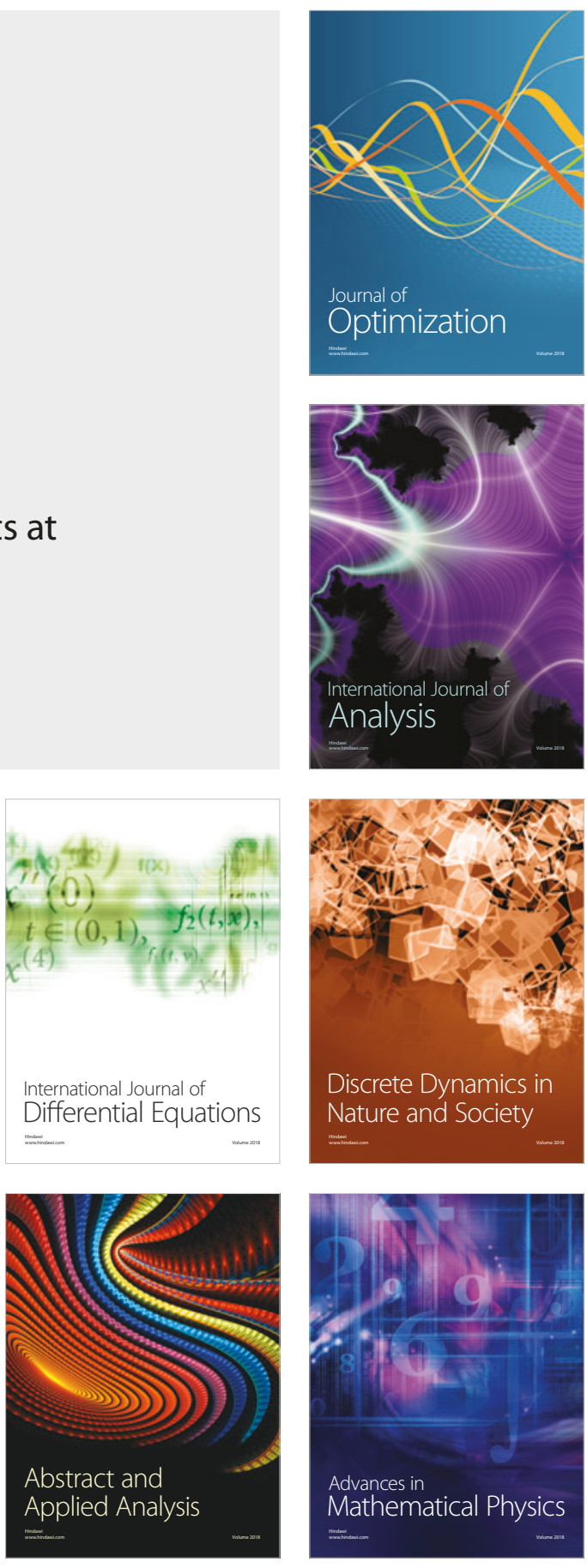